\title{
QED radiative corrections to chiral magnetic effect
}

\author{
Bo Feng, ${ }^{1}$ De-fu Hou, ${ }^{2, *}$ and Hai-cang Ren ${ }^{3,2}$ \\ ${ }^{1}$ School of Physics, Huazhong University of Science and Technology, Wuhan 430074, China \\ ${ }^{2}$ Institute of Particle Physics, Huazhong Normal University, Wuhan 430079, China \\ ${ }^{3}$ Physics Department, The Rockefeller University, 1230 York Avenue, New York,
} New York 10021-6399, USA

(Received 23 October 2018; published 14 February 2019)

\begin{abstract}
We study in this paper the radiative corrections to chiral magnetic current at both zero and nonzero temperature. Our motivation is a radiative correction to the matrix element of the anomalous Ward identity in massless QED stemming from a three-loop diagram where the two photons coming from the one-loop anomalous triangle are rescattered. Through the interplay between the Ward identity and the infrared subtlety of the fermion loop integral, we are able to reproduce the corrections known in literature in a simpler approach and obtain its contribution to the chiral magnetic current at zero temperature. At a nonzero temperature, the infrared subtlety disappeared in a static magnetic field and the three-loop diagram does not contribute to the chiral magnetic current any more. The generalization to all orders of the massless QED and the QCD corrections is discussed.
\end{abstract}

DOI: 10.1103/PhysRevD.99.036010

\section{INTRODUCTION}

Anomaly-induced transport phenomena in systems with chiral fermions have attracted wide interests ranging from high-energy physics to condensed matter physics [1]. One simple anomalous transport phenomenon induced by chiral anomaly is the chiral magnetic effect(CME) [2-5], which predicts charge asymmetries in the final stage of the relativistic heavy ion collisions [6-10] and negative magnetoresistance in some Weyl semimetals $[11,12]$. While there are evidences of CME in condensed matter physics, on the heavy ion collision side, it remains to exclude the noisy backgrounds in order to nail down the real CME signal [13-15].

The chirality imbalance necessary for implementing CME in high-energy physics is provided by the axial charge fluctuations, which is induced by the tunneling among topologically inequivalent gluon configurations of quantum chromodynamics (QCD) at high temperature. In spite of the difficulty in a systematic study of the creation process of the chirality imbalance in the early stage of heavy ion collisions and the subsequent generation of CME in a transient magnetic field, one can model the chirality

\footnotetext{
Corresponding author. houdf@mail.ccnu.edu.cn

Published by the American Physical Society under the terms of the Creative Commons Attribution 4.0 International license. Further distribution of this work must maintain attribution to the author(s) and the published article's title, journal citation, and DOI. Funded by SCOAP ${ }^{3}$.
}

imbalance by a nonzero axial chemical potential $\mu_{5}$, which corresponds to the temporal component of an external axial vector field. To the first order in an external electromagnetic potential $A_{\mu}$ and a constant $\mu_{5}$, the chiral magnetic current can be written as $[4,16]$

$$
J_{i}(q)=\eta \mu_{5} K_{i j}(q) A_{j}(q)
$$

where the factor $\eta=1$ for electrons and $\eta=3 \sum_{f} q_{f}$ for quark-gluon plasma (QGP) with $q_{f}$ the electric charge quanta of the quark flavor $f$. In terms of the AVV threepoint function, $\Lambda_{\mu \nu \rho}\left(Q_{1}, Q_{2}\right)$, standing for the proper vertex of two external photons and an external axial vector field with outgoing photon momenta $Q_{1}$ and $Q_{2}$, the kernel $K_{i j}(q)=\Lambda_{i j 4}(q,-q)$ corresponding to a constant temporal component of the external axial vector field. The lowest order of $\Lambda_{\mu \nu \rho}\left(Q_{1}, Q_{2}\right)$, denoted by $\Delta_{\mu \nu \rho}\left(Q_{1}, Q_{2}\right)$, is represented by the one-loop triangle diagrams. Therefore, it is tempting to relate the CME kernel $K_{i j}(q)$ to the axial anomaly via the limiting process,

$$
K_{i j}(q)=-i \lim _{k_{0} \rightarrow 0} \frac{1}{k_{0}}\left(Q_{1}+Q_{2}\right)_{\rho} \Delta_{i j \rho}\left(Q_{1}, Q_{2}\right),
$$

with $\quad Q_{1}=\left(\mathbf{q}, i\left(\omega+k_{0} / 2\right)\right), Q_{2}=\left(-\mathbf{q}, i\left(-\omega+k_{0} / 2\right)\right)$ and $\Delta_{\mu \nu \rho}$ the amplitudes of the triangle diagrams. From the well-known anomalous Ward identity $[17,18]$,

$$
\left(Q_{1}+Q_{2}\right)_{\rho} \Delta_{\mu \nu \rho}\left(Q_{1}, Q_{2}\right)=-i \frac{e^{2}}{2 \pi^{2}} \epsilon_{\mu \nu \alpha \beta} Q_{1 \alpha} Q_{2 \beta}
$$




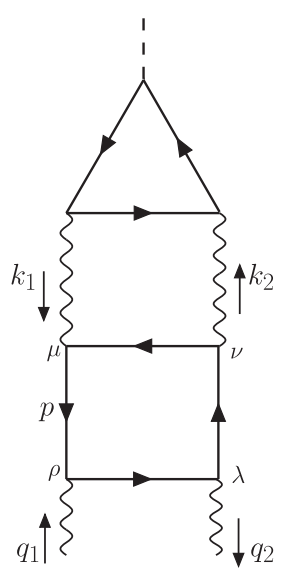

(a)

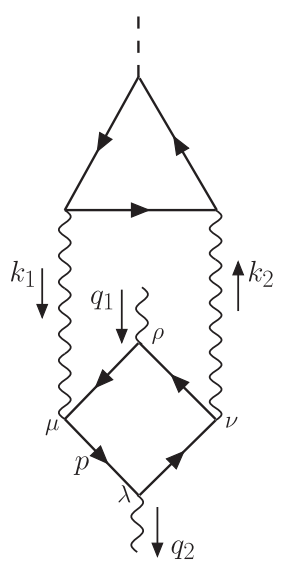

(b)
FIG. 1. The photon-photon scattering contributions to chiral anomaly. The dashed and wavy lines represent the axial-vector and vector fields, respectively.

it follows that

$$
K_{i j}(q)=i \frac{e^{2}}{2 \pi^{2}} \epsilon_{i k j} q_{k},
$$

and the classical form of the chiral magnetic current,

$$
\mathbf{J}=\frac{e^{2}}{2 \pi^{2}} \mu_{5} \mathbf{B}
$$

emerges for one flavor and one color degree of freedom in a static and uniform magnetic field. Because of the nonrenormalization theorem of chiral anomalies, it is expected that Eq. (5) is free from radiative corrections.

Twenty years after the discovery of the nonrenormalization theorem of chiral anomalies, the authors of [19] found a radiative correction to (3) for massless QED, which is UV divergent. This correction is contributed by the three-loop diagram in Fig. 1, where the two photons from the upper triangle are rescattered and the anomalous Ward identity is then modified to the form

$$
\begin{aligned}
\left(Q_{1}+Q_{2}\right)_{\rho} \Lambda_{\mu \nu \rho}\left(Q_{1}, Q_{2}\right)= & -i \frac{e^{2}}{2 \pi^{2}} \epsilon_{\mu \nu \alpha \beta} Q_{1 \alpha} Q_{2 \beta} \\
& \times\left(1-\frac{3 e^{4}}{64 \pi^{4}} \log \frac{\Lambda^{2}}{k^{2}}\right),
\end{aligned}
$$

with $\Lambda$ the ultraviolet cutoff and $k$ the infrared cutoff depending on $Q_{1}$ and $Q_{2}$. This observation is non-trivial in heavy ion collisions, wherein the light $u$ and $d$ quarks can be approximately regarded as massless and (6) generates a radiative correction to the expected chiral magnetic current via

$$
K_{i j}(q)=i \frac{e^{2}}{2 \pi^{2}} \mu_{5} \epsilon_{i k j} q_{k}\left(1-\frac{3 e^{4}}{64 \pi^{4}} \log \frac{\Lambda^{2}}{q^{2}}\right),
$$

where the UV cutoff may be related to an energy scale when the chemical potential description of the axial charge imbalance breaks down. In this paper, we shall calculate the contribution from the lowest-order photon-photon scattering diagrams as in Fig. 1 to the chiral magnetic current in massless fermion case at zero temperature and a nonzero temperature. At zero temperature, we are able to re-produce the $e^{6}$ terms on rhs of (6) following a simpler approach. Our method is based on the interplay between the vector Ward identities and the infrared behavior of the four-photon box diagrams and can be applied to the nonzero temperature case as well.

On the other hand, the constant $\mu_{5}$ limit in (2) becomes subtle at a nonzero temperature. For more general $Q_{1}$ and $Q_{2}$, say $Q_{1}=\left(\mathbf{q}+\mathbf{k} / 2, i\left(\omega+k_{0} / 2\right)\right)$ and $Q_{1}=(-\mathbf{q}+\mathbf{k} / 2$, $\left.i\left(-\omega+k_{0} / 2\right)\right)$ with $k=\left(\mathbf{k}, i k_{0}\right)$ the 4-momentum carried by $\mu_{5}$, the limits $\mathbf{k} \rightarrow 0$ and $k_{0} \rightarrow 0$ do not commute as a symptom of the Lorentz symmetry breaking. To one-loop order, we have $[4,16]$

$\lim _{k_{0} \rightarrow 0} \lim _{\mathbf{k} \rightarrow 0} \Lambda_{i j 4}\left(Q_{1}, Q_{2}\right)=i \frac{e^{2}}{2 \pi^{2}} \mu_{5} \epsilon_{i k j} q_{k}\left[1+\mathcal{O}\left(\frac{\mathbf{q}^{2}}{T^{2}}\right)\right]$

but

$$
\lim _{\mathbf{k} \rightarrow 0} \lim _{k_{0} \rightarrow 0} \Lambda_{i j 4}\left(Q_{1}, Q_{2}\right)=i \frac{e^{2}}{2 \pi^{2}} \mu_{5} \epsilon_{i k j} q_{k} \times \mathcal{O}\left(\frac{\mathbf{q}^{2}}{T^{2}}\right) .
$$

To assess the contribution of the three-loop diagram Fig. 1 at a finite temperature, both orders of limits have to be considered. In order to take the zero energy limit, one has to start with a real time Green's function, whose infrared behavior is more convoluted to track. Fortunately, after taking the limit (8) or (9) of the top triangle in Fig. 1, the real time formulation can be converted into Matsubara formulation for a static magnetic field, where the infrared behavior is transparent, and the same form of the limits (8) or (9) for the summation of the one-loop and three-loop diagrams emerges. In another word, the term on the rhs of (8) that is linear in $\mathbf{q}$ is not subject to the radiative corrections of Fig. 1 and the classical formula (5) is intact. The limits in (8) and (9) correspond to the homogeneous and static limit of the axial chemical potential, respectively. Unlike the ambiguity of the static and uniform magnetic field limit reported in the [5], which can be removed by dressing the fermion propagator [20], the difference between different orders of limit in (8) and (9) stays in higher orders. As is shown in [16], the limit in (8) follows from the nonrenormalization of the axial anomaly while the limit in (9) is the consequence of the vector current conservation if all IR divergence can be removed.

The rest of the paper is organized as follows: in Sec. II, we calculate the radiative correction from Fig. 1 at zero temperature. In Sec. III, the contributions from Fig. 1 at finite temperature are discussed. Section IV is devoted to 
the conclusion along with some open issues. Some technical calculation details and useful proofs are presented in the appendices. Throughout the paper, we will work with Euclidean signature and a 4-momentum is represented by $q_{\mu}=\left(\mathbf{q}, i q_{0}\right)$ with $q_{0}$ the real energy. All gamma matrices in this paper are Hermitian.

\section{ZERO-TEMPERATURE ANALYSIS}

To assess higher-order contributions, it is convenient to distinguish the anomalous Ward identity at the operator level and its matrix elements. In case of electron-photon or quark-photon system, the diagram of the AVV proper vertex can be decomposed into a ladder of two-photon irreducible parts linked by two photon lines shown in Fig. 2, where a photon line stands for a dressed photon propagator. The Anomalous Ward identity at the operator level reads

$$
\partial^{\mu} j_{\mu}^{5}=2 i m j^{5}+i \frac{\alpha_{0}}{4 \pi} \epsilon_{\rho \sigma \lambda \nu} F_{\rho \sigma} F_{\lambda \nu} .
$$

with $\alpha_{0}$ the bare coupling constant, $F_{\rho \sigma}$ the electromagnetic field strength tensor, $j_{\mu}^{5}=i \bar{\psi} \gamma_{\mu} \gamma_{5} \psi$ and $j^{5}=\bar{\psi} \gamma \gamma_{5} \psi$. The operator anomaly corresponds to the 4-divergence with respect to the axial vector vertex of the shaded triangle at the leftmost in Fig. 2, whose anomaly term on rhs is free from radiative corrections as proved by Adler and Bardeen [18]. In another word, besides the one-loop triangle, the fermion loop with the axial vector vertex of all other diagrams included in the shaded triangle have more than two photon vertices so the shift of loop momentum does not generate extra terms. The entire diagram is obtained by taking the matrix element of Eq. (10) between the vacuum and a state with two photons of momenta $\left(Q_{1}, Q_{2}\right)$ and polarizations $(\rho, \lambda)$. The matrix element takes the form
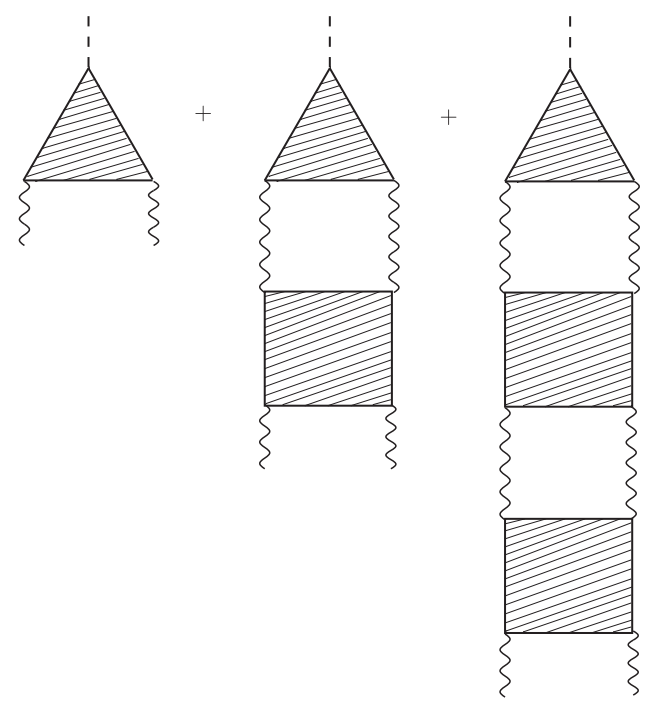

FIG. 2. The two-photon reducible AVV diagrams. The shaded box and triangle represent the two-photon irreducible diagrams.

$$
\begin{aligned}
\left(Q_{1}+Q_{2}\right)_{\mu} \Lambda_{\mu \rho \lambda}\left(Q_{1}, Q_{2}\right) \\
=-i\left[2 m G\left(\frac{Q_{1}^{2}}{m^{2}}, \frac{Q_{2}^{2}}{m^{2}}, \frac{Q_{1} \cdot Q_{2}}{m^{2}}\right)+H\left(\frac{Q_{1}^{2}}{m^{2}}, \frac{Q_{2}^{2}}{m^{2}}, \frac{Q_{1} \cdot Q_{2}}{m^{2}}\right)\right] \\
\quad \times \epsilon_{\rho \lambda \alpha \beta} Q_{1 \alpha} Q_{2 \beta},
\end{aligned}
$$

with $G$ corresponding to the naive divergence and $H$ to the anomaly coefficient. The Adler-Bardeen theorem [18] states that the naive divergence $2 m j^{5}$ for a nonzero mass has a known value to all orders at a particular kinematic point where external momenta vanish, i.e.,

$2 m G(0,0,0)=-H(0,0,0), \quad$ and $\quad H(0,0,0)=\frac{2 \alpha}{\pi}$,

with $\alpha$ the renormalized coupling constant. The first equation above follows from the Sutherland-Veltman theorem [21] and the second one states that the photon-photon scattering contribution in Fig. 1 vanishes at this kinematic point. On the contrary, however, the kinematic point that validates the Adler-Bardeen theorem cannot be attained if zero fermion mass limit is taken first, which renders the arguments of $G$ and $H$ approaching infinity. Therefore, the discovery that the matrix element of the anomaly, $H$, acquires a radiative correction is not surprising, nor does it invalidate the Adler-Bardeen theorem [22].

Both the Sutherland-Veltman theorem and AdlerBardeen theory follow from the Ward identity of the electromagnetic gauge invariance. In the rest of this section, we shall show that the Ward identity argument failed in the presence of the infrared divergence pertaining to massless fermions. Our analysis also gives rise to a much simpler derivation of the radiative correction in (6). A more pedagogical evaluation of the diagrams in Fig. 1 can be found in Appendix A. To make the infrared singularities transparent, we shall work with imaginary energy formulation for the rest of this section. This amounts to a Wick rotation of all 4-momenta (external and internal). The real energy result can be obtained by undoing the Wick rotation of the external momenta appropriately.

It follows from (3) that the divergence with respect to the axial vector vertex of the top triangle in Fig. 1 can be replaced by a two photon vertex specified by the rhs of (3). The three-loop contribution to the 4-divergence $\left(Q_{1}+\right.$ $\left.Q_{2}\right)_{\mu} \Lambda_{\mu \rho \lambda}\left(Q_{1}, Q_{2}\right)$ of (11) is thereby reduced to a two-loop diagram whose amplitude reads

$\Lambda_{\rho \lambda}(q, Q)=i \frac{e^{6}}{2 \pi^{2}} \epsilon_{\mu \nu \alpha \beta} Q_{\alpha} \int \frac{d^{4} k}{(2 \pi)^{4}} \frac{k_{\beta}}{\left(k^{2}\right)^{2}} \Gamma_{\mu \nu \rho \lambda}(q, q ; k)$,

to the leading order in $Q$, where $Q_{1}=-q+Q / 2, Q_{2}=$ $q+Q / 2$ and $\Gamma_{\mu \nu \rho \lambda}\left(q_{1}, q_{2} ; k_{1}\right)$ is the amplitude of the box diagram of four photons in Fig. 3. We have 


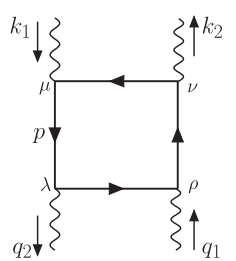

$(I)$

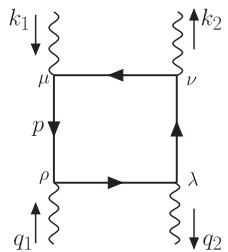

$(I V)$

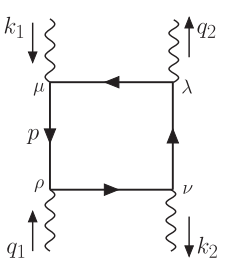

$(I I)$

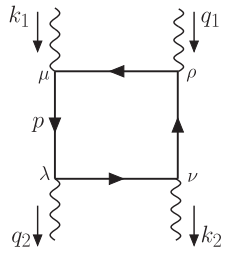

$(V)$

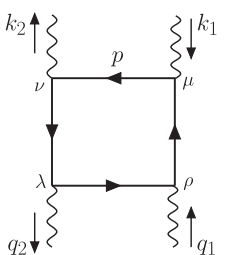

$(I I I)$

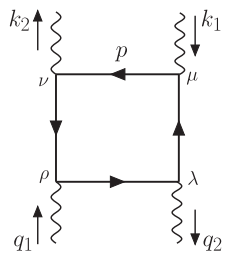

$(V I)$
FIG. 3. The four-photon box diagrams including all permutations.

$$
\Gamma_{\mu \nu \rho \lambda}\left(q_{1}, q_{2} ; k_{1}\right)=\mathrm{I}+\mathrm{II}+\mathrm{III}+\mathrm{IV}+\mathrm{V}+\mathrm{VI},
$$

with

$$
\begin{aligned}
& \mathrm{I}=-\int \frac{d^{4} p}{(2 \pi)^{4}} \operatorname{tr} \gamma_{\mu} \frac{1}{\not p-\not \ell_{1}} \gamma_{\nu} \frac{1}{\not p-\not q_{2}+\not \not_{1}} \gamma_{\rho} \frac{1}{\not p-\not q_{2}} \gamma_{\lambda} \frac{1}{\not p}, \\
& \mathrm{II}=-\int \frac{d^{4} p}{(2 \pi)^{4}} \operatorname{tr} \gamma_{\mu} \frac{1}{\not p-\not l_{1}} \gamma_{\lambda} \frac{1}{\not p-\not \ell_{1}+\not d_{2}} \gamma_{\nu} \frac{1}{\not p+\not q_{1}} \gamma_{\rho} \frac{1}{\not p} \text {, } \\
& \mathrm{III}=-\int \frac{d^{4} p}{(2 \pi)^{4}} \operatorname{tr} \gamma_{\mu} \frac{1}{\not p} \gamma_{\rho} \frac{1}{\not p-\not \not_{1}} \gamma_{\lambda} \frac{1}{\not p-\not \not_{1}+\not \not_{2}} \gamma_{\nu} \frac{1}{\not p+\not l_{1}} \text {, } \\
& \mathrm{IV}=-\int \frac{d^{4} p}{(2 \pi)^{4}} \operatorname{tr} \gamma_{\mu} \frac{1}{\not p-\not \ell_{1}} \gamma_{\nu} \frac{1}{\not p-\not \not_{2}+\not \not_{1}} \gamma_{\lambda} \frac{1}{\not p+\not \phi_{1}} \gamma_{\rho} \frac{1}{\not p} \text {, } \\
& \mathrm{V}=-\int \frac{d^{4} p}{(2 \pi)^{4}} \operatorname{tr} \gamma_{\mu} \frac{1}{\not p} \gamma_{\rho} \frac{1}{\not p-\not \not_{1}} \gamma_{\nu} \frac{1}{\not p+\not \ell_{1}-\not \not_{2}} \gamma_{\lambda} \frac{1}{\not p+\not k_{1}} \text {, } \\
& \mathrm{VI}=-\int \frac{d^{4} p}{(2 \pi)^{4}} \operatorname{tr} \gamma_{\mu} \frac{1}{\not p} \gamma_{\lambda} \frac{1}{\not p+\not q_{2}} \gamma_{\rho} \frac{1}{\not p-\not \not_{1}+\not q_{2}} \gamma_{\nu} \frac{1}{\not p+\not \ell_{1}} \text {. }
\end{aligned}
$$

It is well known that $\Gamma_{\mu \nu \rho \lambda}\left(q_{1}, q_{2} ; k_{1}\right)$ is UV convergent because of the gauge invariance. A regulator is yet required to maintain the gauge invariance in general since the individual terms of (15) remain logarithmically divergent. To see this, let us contract a photon vertex with the 4-momentum of the photon, say, $q_{1 \rho} \Gamma_{\mu \nu \rho \lambda}\left(q_{1}, q_{2} ; k_{1}\right)$. Following the standard textbook approach [23], the integrand of $q_{1 \rho} \Gamma_{\mu \nu \rho \lambda}\left(q_{1}, q_{2} ; k_{1}\right)$ can be written as the difference of two terms, differing by the shift of the integration momentum. Each term itself is linearly divergent by power counting and regulator is required to render the shift of the integration momentum legitimate such that the Ward identity holds. On the other hand, upon contracting with the epsilon tensor from the anomaly, each term in the difference of the integrand of $\epsilon_{\mu \nu \alpha \beta} q_{1 \rho} \Gamma_{\mu \nu \rho \lambda}\left(q_{1}, q_{2} ; k_{1}\right)$ becomes logarithmic divergent and the regulator is no longer required to maintain the Ward identity (see Appendix B for details). Also, the difference from shifting the integration variable by an external momentum in the integrand of $q_{1 \rho} \Gamma_{\mu \nu \rho \lambda}\left(q_{1}, q_{2} ; k_{1}\right)$ can be Taylor expanded in the powers of the external momenta, leaving the integrand a total derivative with respect to the integration momentum.

It follows that

$$
\begin{aligned}
\mathrm{I}+\mathrm{II}+\mathrm{IV} & =i \int \frac{d^{4} p}{(2 \pi)^{4}} \frac{\partial}{\partial p_{\lambda}} \operatorname{tr} \gamma_{\mu} \frac{1}{\not p-\not \ell_{1}} \gamma_{\nu} \frac{1}{\not p+\not \not_{1}} \gamma_{\rho} \frac{1}{\not p}, \\
\mathrm{III}+\mathrm{V}+\mathrm{VI} & =i \int \frac{d^{4} p}{(2 \pi)^{4}} \frac{\partial}{\partial p_{\lambda}} \operatorname{tr} \gamma_{\mu} \frac{1}{\not p} \gamma_{\rho} \frac{1}{\not \not p-\not \phi_{1}} \gamma_{\nu} \frac{1}{\not p+\not l_{1}},
\end{aligned}
$$

at $q_{2}=0$ and

$\mathrm{I}+\mathrm{IV}+\mathrm{V}$

$=i \int \frac{d^{4} p}{(2 \pi)^{4}} \frac{\partial}{\partial p_{\rho}} \operatorname{tr} \gamma_{\mu} \frac{1}{\not p-\not \ell_{1}} \gamma_{\nu} \frac{1}{\not p-\not q_{2}} \gamma_{\lambda} \frac{1}{\not \not p}+\Xi_{\mu \nu \rho \lambda}$,

$\mathrm{II}+\mathrm{III}+\mathrm{VI}$

$=i \int \frac{d^{4} p}{(2 \pi)^{4}} \frac{\partial}{\partial p_{\rho}} \operatorname{tr} \gamma_{\mu} \frac{1}{\not p} \gamma_{\lambda} \frac{1}{\not p+\not q_{2}} \gamma_{\nu} \frac{1}{\not p+\not \not_{1}}+\Xi_{\mu \nu \rho \lambda}^{\prime}$,

at $q_{1}=0$, where

$$
\Xi_{\mu \nu \rho \lambda}=\int \frac{d^{4} p}{(2 \pi)^{4}} \operatorname{tr} \gamma_{\mu}\left(\frac{1}{\not p-\not \not_{1}} \gamma_{\rho} \frac{1}{\not p-\not \not_{1}} \gamma_{\nu} \frac{1}{\not p-\not q_{2}} \gamma_{\lambda} \frac{1}{\not p}-\frac{1}{\not p} \gamma_{\rho} \frac{1}{\not p} \gamma_{\nu} \frac{1}{\not p+\not k_{1}-\not \not_{2}} \gamma_{\lambda} \frac{1}{\not p+\not k_{1}}\right)=0,
$$

and

$$
\Xi_{\mu \nu \rho \lambda}^{\prime}=\int \frac{d^{4} p}{(2 \pi)^{4}} \operatorname{tr} \gamma_{\mu}\left(\frac{1}{\not p} \gamma_{\lambda} \frac{1}{\not p+\not \not_{2}} \gamma_{\nu} \frac{1}{\not p+\not k_{1}} \gamma_{\rho} \frac{1}{\not p+\not \not_{1}}-\frac{1}{\not p-\not \not_{1}} \gamma_{\lambda} \frac{1}{\not p-\not \not_{1}+\not q_{2}} \gamma_{\nu} \frac{1}{\not p} \gamma_{\rho} \frac{1}{\not p}\right)=0
$$

upon shifting the integration momentum, and we end up with the total derivative forms of the integrands of $\Gamma_{\mu \nu \rho \lambda}\left(q_{1}, 0 ; k_{1}\right)$ and $\Gamma_{\mu \nu \rho \lambda}\left(0, q_{2} ; k_{1}\right)$. In particular, 


$$
\begin{aligned}
\epsilon_{\mu \nu \alpha \beta} \Gamma_{\mu \nu \rho \lambda}\left(0,0 ; k_{1}\right) & =i \int \frac{d^{4} p}{(2 \pi)^{4}} \frac{\partial}{\partial p_{\lambda}} \epsilon_{\mu \nu \alpha \beta} \operatorname{tr} \gamma_{\mu}\left(\frac{1}{\not p-\not k_{1}} \gamma_{\nu} \frac{1}{\not p} \gamma_{\rho} \frac{1}{\not p}+\frac{1}{\not p} \gamma_{\rho} \frac{1}{\not p} \gamma_{\nu} \frac{1}{\not p+\not \not_{1}}\right), \\
& =i \int \frac{d^{4} p}{(2 \pi)^{4}} \frac{\partial}{\partial p_{\rho}} \epsilon_{\mu \nu \alpha \beta} \operatorname{tr} \gamma_{\mu}\left(\frac{1}{\not p-\not \not_{1}} \gamma_{\nu} \frac{1}{\not p} \gamma_{\lambda} \frac{1}{\not p}+\frac{1}{\not p} \gamma_{\lambda} \frac{1}{\not p} \gamma_{\nu} \frac{1}{\not p+\not l_{1}}\right) .
\end{aligned}
$$

While we tentatively employ the Stokes theorem to convert the volume integral into a surface integral at infinity of the momentum space, care must be exercised because of the UV and IR behavior of the integrand. Introducing a sphere of radius $\epsilon$ around each point of IR singularity, i.e., $p=0$ and $p= \pm k_{1}$, we apply the Stokes theorem outside the spheres. The expressions under each partial derivative vanish faster than $p^{-3}$ as $p \rightarrow \infty$. So the surface integral at infinity does not contribute, and we are left with the surface integral of each sphere as well as the volume integral over the interior of them. The integrand of the surface integral grows like $p^{-2}$ or $\left(p \mp k_{1}\right)^{-1}$, while the corresponding volume integral grows like $p^{-3}$ or $\left(p \mp k_{1}\right)^{-2}$. Consequently, these integrals vanish as $\epsilon \rightarrow 0$, and we obtain that

$$
\epsilon_{\mu \nu \alpha \beta} \Gamma_{\mu \nu \rho \lambda}(0,0, k)=0 .
$$

Taking the derivatives of (16) with respect to $q_{1}$, and (17) with respect to $q_{2}$, we obtain that

$$
\left.\frac{\partial}{\partial q_{1 \sigma}} \Gamma_{\mu \nu \rho \lambda}\left(q_{1}, 0 ; k\right)\right|_{q_{1}=0}=\int \frac{d^{4} p}{(2 \pi)^{4}} \frac{\partial}{\partial p_{\lambda}} \operatorname{tr} \gamma_{\mu}\left(-\frac{1}{\not p-\not k} \gamma_{\nu} \frac{1}{\not p} \gamma_{\sigma} \frac{1}{\not p} \gamma_{\rho} \frac{1}{\not p}+\frac{1}{\not p} \gamma_{\rho} \frac{1}{\not p} \gamma_{\sigma} \frac{1}{\not p} \gamma_{\nu} \frac{1}{\not p+\not k}\right),
$$

and

$$
\left.\frac{\partial}{\partial q_{2 \sigma}} \Gamma_{\mu \nu \rho \lambda}\left(0, q_{2} ; k\right)\right|_{q_{2}=0}=\int \frac{d^{4} p}{(2 \pi)^{4}} \frac{\partial}{\partial p_{\rho}} \operatorname{tr} \gamma_{\mu}\left(\frac{1}{\not p-\not h} \gamma_{\nu} \frac{1}{\not p} \gamma_{\sigma} \frac{1}{\not \not p} \gamma_{\lambda} \frac{1}{\not p}-\frac{1}{\not p} \gamma_{\lambda} \frac{1}{\not p} \gamma_{\sigma} \frac{1}{\not p} \gamma_{\nu} \frac{1}{\not \supset+\not k}\right) .
$$

It follows from (22) and (23) that the coefficient of the linear term in $q$ of $\Gamma_{\mu \nu \rho \lambda}(q, q, k)$ reads

$$
\begin{aligned}
\left.\frac{\partial}{\partial q_{\sigma}} \Gamma_{\mu \nu \rho \lambda}(q, q ; k)\right|_{q=0} & =\left.\frac{\partial}{\partial q_{1 \sigma}} \Gamma_{\mu \nu \rho \lambda}\left(q_{1}, 0 ; k\right)\right|_{q_{1}=0}+\left.\frac{\partial}{\partial q_{2 \sigma}} \Gamma_{\mu \nu \rho \lambda}\left(0, q_{2} ; k\right)\right|_{q_{2}=0}, \\
& =-\int \frac{d^{4} p}{(2 \pi)^{4}}\left[\frac{\partial}{\partial p_{\lambda}} \operatorname{tr} \gamma_{\mu}\left(\frac{1}{\not \supset-\not h} \gamma_{\nu} \frac{1}{\not p} \gamma_{\sigma} \frac{1}{\not p} \gamma_{\rho} \frac{1}{\not p}-\frac{1}{\not \not p} \gamma_{\rho} \frac{1}{\not p} \gamma_{\sigma} \frac{1}{\not p} \gamma_{\nu} \frac{1}{\not p+\not k}\right)-(\rho \leftrightarrow \lambda)\right] .
\end{aligned}
$$

Let us focus on the integration of the first term inside the parentheses and apply the Stokes theorem outside the small spheres centered at $p=0$ and $p=k$ as before. The surface integral at infinity drops, and the singularity at $p=k$ is too weak to contribute. The integration around the singularity $p=0$, however, has to be retained. Denoting the small sphere centered at the origin by $B_{\epsilon}$ and its surface by $\partial B_{\epsilon}$, we end up with

$$
\begin{aligned}
J_{\mu \nu \rho \lambda \sigma} & \equiv-\int \frac{d^{4} p}{(2 \pi)^{4}} \frac{\partial}{\partial p_{\lambda}} \operatorname{tr} \gamma_{\mu} \frac{1}{\not p-\not h} \gamma_{\nu} \frac{1}{\not p} \gamma_{\sigma} \frac{1}{\not p} \gamma_{\rho} \frac{1}{\not p}, \\
& =\frac{1}{(2 \pi)^{4}} \int_{\partial B_{\varepsilon}} d^{3} \hat{p}_{\lambda} \frac{\operatorname{tr} \gamma_{\mu}(\not \not-\not h) \gamma_{\nu} \hat{p} \gamma_{\sigma} \hat{p} \gamma_{\rho} \hat{p}}{(p-k)^{2}}-\int_{B_{\varepsilon}} \frac{d^{4} p}{(2 \pi)^{4}} \frac{\partial}{\partial p_{\lambda}} \operatorname{tr} \gamma_{\mu} \frac{1}{\not p-\not k} \gamma_{\nu} \frac{1}{\not p} \gamma_{\sigma} \frac{1}{\not p} \gamma_{\rho} \frac{1}{\not \not p},
\end{aligned}
$$

where $\hat{p}$ denotes the unit vector in the direction of $p$. As $\epsilon \rightarrow 0, p-k \rightarrow-k$, and

$$
\begin{aligned}
\epsilon_{\mu \nu \alpha \beta} Q_{\alpha} k_{\beta} J_{\mu \nu \rho \lambda \sigma}= & -\frac{1}{8 \pi^{2} k^{2}} \epsilon_{\mu \nu \alpha \beta} Q_{\alpha} k_{\beta}\left\langle\hat{p}_{\lambda} \operatorname{tr} \gamma_{\mu} \not k \gamma_{\nu} \hat{p} \gamma_{\sigma} \hat{p} \gamma_{\rho} \hat{\boldsymbol{p}}\right\rangle_{p} \\
& +\frac{1}{8 \pi^{2} k^{2}} \epsilon_{\mu \nu \alpha \beta} Q_{\alpha} k_{\beta} \int_{0}^{\epsilon} d p p^{3}\left\langle\frac{\partial}{\partial p_{\lambda}} \frac{\operatorname{tr} \gamma_{\mu} \not k \gamma_{\nu} \not p \gamma_{\sigma} \not p \gamma_{\rho} \not \supset}{\left(p^{2}\right)^{3}}\right\rangle_{p},
\end{aligned}
$$

where $\langle\ldots\rangle_{p}$ denotes the average over the direction of $p$. We have 


$$
\begin{aligned}
\left\langle\hat{p}_{\mu} \hat{p}_{\nu}\right\rangle_{p} & =\frac{1}{4} \delta_{\mu \nu}, \\
\left\langle\hat{p}_{\mu} \hat{p}_{\nu} \hat{p}_{\rho} \hat{p}_{\lambda}\right\rangle_{p} & =\frac{1}{24}\left(\delta_{\mu \nu} \delta_{\rho \lambda}+\delta_{\mu \rho} \delta_{\nu \lambda}+\delta_{\mu \lambda} \delta_{\nu \rho}\right) .
\end{aligned}
$$

It is readily seen that the second integral of (26) vanishes because

$$
\left\langle\frac{\partial}{\partial p_{\lambda}} \frac{p_{\mu} p_{\nu} p_{\rho}}{\left(p^{2}\right)^{3}}\right\rangle_{p}=0
$$

and we are left with

$$
\begin{aligned}
\epsilon_{\mu \nu \alpha \beta} Q_{\alpha} k_{\beta} J_{\mu \nu \rho \lambda \sigma}= & -\frac{1}{8 \pi^{2} k^{2}} \epsilon_{\mu \nu \alpha \beta} Q_{\alpha} k_{\beta}\left\langle\hat{p}_{\lambda} \operatorname{tr} \gamma_{\mu} \not k \gamma_{\nu} \hat{p} \gamma_{\sigma} \hat{p} \gamma_{\rho} \hat{\boldsymbol{p}}\right\rangle_{p}, \\
= & \frac{1}{96 \pi^{2} k^{2}} \epsilon_{\mu \nu \alpha \beta} Q_{\alpha} k_{\beta} k_{\tau} \\
& \times \operatorname{tr} \gamma_{\mu} \gamma_{\tau} \gamma_{\nu}\left(\gamma_{\lambda} \gamma_{\sigma} \gamma_{\rho}+\gamma_{\rho} \gamma_{\lambda} \gamma_{\sigma}+\gamma_{\sigma} \gamma_{\rho} \gamma_{\lambda}\right),
\end{aligned}
$$

where the second step follows from (27), and the following two identities of gamma matrices are

$$
\begin{aligned}
\gamma_{\alpha} \gamma_{\mu} \gamma_{\alpha} & =-2 \gamma_{\mu}, \\
\gamma_{\alpha} \gamma_{\mu} \gamma_{\nu} \gamma_{\rho} \gamma_{\alpha} & =-2 \gamma_{\rho} \gamma_{\nu} \gamma_{\mu} .
\end{aligned}
$$

Further reduction is facilitated by the identity

$$
\epsilon_{\mu \nu \alpha \beta} \gamma_{\mu} \gamma_{\tau} \gamma_{\nu}=2\left(\delta_{\tau \alpha} \gamma_{\beta}-\delta_{\tau \beta} \gamma_{\alpha}\right) \gamma_{5},
$$

and we obtain that

$$
\begin{aligned}
\epsilon_{\mu \nu \alpha \beta} Q_{\alpha} k_{\beta} J_{\mu \nu \rho \lambda \sigma} & =\frac{1}{16 \pi^{2} k^{2}}\left(k \cdot Q k_{\alpha}-k^{2} Q_{\alpha}\right) \operatorname{tr} \gamma_{5} \gamma_{\sigma} \gamma_{\rho} \gamma_{\lambda} \gamma_{\alpha}, \\
& =\frac{1}{4 \pi^{2} k^{2}} \epsilon_{\sigma \rho \lambda \alpha}\left(k \cdot Q k_{\alpha}-k^{2} Q_{\alpha}\right) .
\end{aligned}
$$

It is straightforward to show that all four terms of the integrand of (24) contribute equally, i.e.,

$$
\left.\frac{\partial}{\partial q_{\sigma}} \Gamma_{\mu \nu \rho \lambda}(q, q ; k)\right|_{q=0}=4 J_{\mu \nu \rho \lambda \sigma},
$$

and the Taylor expansion of $\epsilon_{\mu \nu \alpha \beta} Q_{\alpha} k_{\beta} \Gamma_{\mu \nu \rho \lambda}(q, q ; k)$ to the linear order in $q$ reads

$\epsilon_{\mu \nu \alpha \beta} Q_{\alpha} k_{\beta} \Gamma_{\mu \nu \rho \lambda}(q, q ; k)=\frac{1}{\pi^{2} k^{2}} q_{\sigma} \epsilon_{\sigma \rho \lambda \alpha}\left(k \cdot Q k_{\alpha}-k^{2} Q_{\alpha}\right)$,

following (21), (33), and (32). Substituting (34) into (13), the integration over $k$ diverges logarithmically in both UV and IR regions. The former is genuine and its coefficient matches that of $\ln \Lambda^{2}$ in (6). The infrared divergence, however, is caused by the inappropriate small $q$ expansion as $k \rightarrow 0$. It follows from (15) that $\Gamma_{\mu \nu \rho \lambda}(q, q ; k)$ tends to a finite limit as $k \rightarrow 0$ with $q$ in the denominator of the integrand, which serves as an infrared cutoff. The radiative corrections in (6) are thereby reproduced without tedious multiloop calculations. The finite term pertaining to the logarithm cannot be obtained that simply, as is the case with the multiloop calculation in [19]. Note that the authors of $[17,18]$ argued that this divergence could be removed through the renormalization of the axial vector vertex. This, however, may not be the case. As we have shown explicitly, the logarithmic divergence is contributed by both diagrams in Fig. 1, and the second diagram does not contain a decorated axial vertex with two fermion legs. Other alternatives need to be explored. One possible mechanism is the wave function renormalization of the axial vector field, which corresponds to the renormalization of the axial chemical potential $\mu_{5}$ in case of CME.

Note that the same diagrams as those in Fig. 1 with the two internal photon lines replaced by gluon lines may contribute to the chiral magnetic current more significantly because of the stronger coupling strength. The kernel of the chiral magnetic current in this case becomes [24]

$$
K_{i j}(q)=i \frac{e^{2}}{2 \pi^{2}} \mu_{5} \epsilon_{i k j} q_{k}\left(1-\frac{g^{4}}{32 \pi^{4}} \log \frac{\Lambda^{2}}{q^{2}}\right),
$$

with $g$ the QCD coupling constant.

\section{FINITE-TEMPERATURE ANALYSIS}

In this section, we shall discuss whether the same radiative corrections to the CME current from the twophoton reducible diagrams remain at a nonzero temperature. The problem is subtle because of the Lorentz symmetry breaking at a nonzero temperature, which may cause the limit $q \rightarrow 0$ of the 4-momentum $q$ flowing in a vertex to be ambiguous. That is, $\lim _{q_{0} \rightarrow 0} \lim _{|\mathbf{q}| \rightarrow 0}$ and $\lim _{|\mathbf{q}| \rightarrow 0} \lim _{q_{0} \rightarrow 0}$ may not agree. The ambiguity already shows up at one-loop calculations of CME current with $q$ the 4-momentum entering the vertex of the axial chemical potential [16] or the vertex attached to the external magnetic field [5]. In the following, we shall examine the two orders of limit separately for the former case. To carry out the zero energy limit involved, one has to work with a real time formulation, of which the closed-time-path (CTP) Green's functions [25] serve our purpose. As in the case of zero temperature, we designate $q_{1}$ to the incoming 4-momentum of the external vector potential and $q_{2}$ to the outgoing momentum of the electric current. The incoming 4-momentum of the axial chemical potential is then $Q=q_{2}-q_{1}$.

\section{A. The CTP Green's functions}

The CTP Green's functions are generated by a path integral whose action is the integration of the classical 
Lagrangian along a closed time path which consists of a forward branch, $\int_{-\infty}^{\infty} d t(\ldots)$ and a backward branch, $\int_{\infty}^{-\infty} d t(\ldots)$. The number of degrees of freedom is thereby doubled. Explicitly, the path integral that generates the CTP Green's function is given by

$$
\begin{aligned}
Z_{\mathrm{CTP}}= & \int\left[d \phi_{1}(t)\right]\left[d \phi_{2}(t)\right] e^{i S_{\mathrm{CTP}}\left[\phi_{1}(t), \phi_{2}(t)\right]} \\
& \times W\left[\phi_{2}(-\infty), \phi_{1}(-\infty)\right]
\end{aligned}
$$

with the action

$$
S_{\mathrm{CTP}}\left[\phi_{1}(t), \phi_{2}(t)\right]=\int_{-\infty}^{\infty} d t L_{\mathrm{cl} .}\left[\phi_{1}(t)\right]+\int_{\infty}^{-\infty} d t L_{\mathrm{cl} .}\left[\phi_{2}(t)\right],
$$

where $\phi$ stands for the set of all field variables and

$$
W\left[\phi^{\prime}, \phi\right]=\left\langle\phi^{\prime}|\rho| \phi\right\rangle
$$

generates initial correlations with $\rho$ a density operator. As the path integral for the ordinary Feynman diagrams, the interaction is adiabatically switched on since $t=-\infty$ along the forward time branch and is adiabatically switched off towards $t=-\infty$ along the backward branch. The timeordering operator underlying the CTP Green's functions becomes the path-ordering along the closed time path, i.e., ordinary time ordering along the forward branch and antitime ordering along the backward branch with the backward branch preceding the forward branch. While the CTP Green's function can be described by the same set of Feynman diagrams, the number of components of an $n$ point CTP Green's function is $2^{n}$ times of the corresponding Green's function of the Feynman because of the additional CTP indices pertaining to external lines. It follows from (37) that a bare CTP vertex is formed by the field variables along the same time branch and carries one CTP index, while an internal line, a CTP propagator, carries two CTP indices and has four components. All CTP indices not attached to external lines have to be summed. Given all eight CTP components of the diagram for the chiral magnetic effect, the electric current in response to an external magnetic field and an axial chemical potential is obtained by fixing the CTP index of the current vertex on the forward time branch and summing up all CTP indices of the magnetic field and axial chemical potential vertices, which take the same values along both time branches. A photon vertex in CTP formulation reads $\gamma_{\mu}\left(-\gamma_{\mu}\right)$ on the forward (backward) time branch with the minus sign taking into account the opposite direction of the time integration in (37). The axial vector vertex is similar, i.e., $\gamma_{\mu} \gamma_{5}$ and $-\gamma_{\mu} \gamma_{5}$. With an equilibrium density matrix at $t=-\infty, \rho \propto e^{-\beta H_{0}}$ with $H_{0}$ the free Hamiltonian, the four CTP components
$S_{a b}(p \mid m)$ of a free Dirac propagator of mass $m$ takes the form

$$
\begin{aligned}
S_{11}(p \mid m)= & \frac{i}{\not p+i 0^{+}-m}-\pi \frac{\not p+m}{E} \\
& \times\left[f(E) \delta\left(p_{0}-E\right)+f(E) \delta\left(p_{0}+E\right)\right], \\
S_{12}(p \mid m)= & -\pi \frac{\not p+m}{E}\left\{f(E) \delta\left(p_{0}-E\right)\right. \\
& \left.+[f(E)-1] \delta\left(p_{0}+E\right)\right\}, \\
S_{21}(p \mid m)= & -\pi \frac{\not p+m}{E}[f(E)-1] \delta\left(p_{0}-E\right) \\
& \left.+f(E) \delta\left(p_{0}+E\right)\right\}, \\
S_{22}(p \mid m)= & \frac{-i}{\not p-i 0^{+}-m}-\pi \frac{\not p+m}{E}\left[f(E) \delta\left(p_{0}-E\right)\right. \\
& \left.+f(E) \delta\left(p_{0}+E\right)\right],
\end{aligned}
$$

where $p=\left(\mathbf{p}, i p_{0}\right), E=\sqrt{\mathbf{p}^{2}+m^{2}}, \not p \equiv-i \gamma_{\nu} p_{\nu}$, and $f(x)=1 /\left(e^{\beta x}+1\right)$ is the Fermi distribution function at temperature $T=1 / \beta$. Let $\Lambda_{\mu \nu \rho}^{a b c}\left(Q_{1}, Q_{2}\right)$ be a CTP component of the three-point function corresponding to $\Lambda_{\mu \nu \rho}\left(Q_{1}, Q_{2}\right)$ at zero temperature with the index pair $(\mu, a)$ associated with the electric current, the index pair $(\nu, b)$ with the external electromagnetic potential, and the index pair $(\rho, c)$ with the axial vector vertex. The retarded Green's function underlying the current in response to an external electromagnetic potential and an external axial vector field reads

$$
\Lambda_{\mu \nu \rho}^{R}\left(Q_{1}, Q_{2}\right)=\sum_{b, c} \Lambda_{\mu \nu \rho}^{1 b c}\left(Q_{1}, Q_{2}\right)
$$

We have $\mu=i, \nu=j, \rho=4, Q_{1}=-q_{1}$, and $Q_{2}=q_{2}$ for the radiative correction to CME by the diagrams in Fig. 1.

After working out the double $\operatorname{limit}_{\lim _{Q_{0} \rightarrow 0}} \lim _{\mathbf{Q} \rightarrow \mathbf{0}}$ or $\lim _{\mathbf{Q} \rightarrow \mathbf{0}} \lim _{Q_{0} \rightarrow 0}$, the rest of the calculation can be greatly simplified for the standard setup of chiral magnetic current, i.e., the spatial current under a static and homogeneous magnetic field. The summation (40) yields the Matsubara amplitude; i.e., the different CTP components in (40) add up to a single term which is described by the same diagram with all continuous energies flowing along internal lines replaced by discrete Matsubara energies. The loopwise proof of this statement is rather tedious. A nonperturbative proof in terms of spectral representations is presented in Appendix C.

\section{B. The order $\lim _{Q_{0} \rightarrow 0} \lim _{\mathrm{Q} \rightarrow 0}$ :}

The first limit, $\mathbf{Q} \rightarrow 0$ renders the axial chemical potential homogeneous, and we have $\mathbf{q}_{1}=\mathbf{q}_{2} \equiv \mathbf{q}$. The CTP formulation has to be employed to evaluate the second limit, $Q_{0} \rightarrow 0$. Let us start with an excursion to prove that 
the divergence of the AVV triangle in CTP formulation can be replaced by a bare CTP vertex, like in the zero temperature case. To evaluate the fermion loop, it is convenient to introduce the matrix notation with CTP indices traced together with the spinor and internal indices. Introducing the $2 \times 2$ matrix with respect to the CTP indices, i.e.,

$$
\eta_{1}=\left(\begin{array}{ll}
1 & 0 \\
0 & 0
\end{array}\right), \quad \text { and } \quad \eta_{2}=\left(\begin{array}{ll}
0 & 0 \\
0 & 1
\end{array}\right)
$$

different CTP components can be projected out with one of them. It can be shown explicitly that

$$
\begin{aligned}
& S(p+q \mid m) \unrhd \gamma_{5} \eta_{c} S(p \mid m) \\
& =i\left[\eta_{c} \gamma_{5} S(p \mid m)+S(p+q \mid m) \gamma_{5} \eta_{c}\right] \\
& \quad+2 m S(p+q \mid m) \Gamma_{5} \eta_{c} S(p \mid m),
\end{aligned}
$$

with

$$
\mathscr{Q} \equiv-i q_{\mu} \Gamma_{\mu}
$$

See Appendix D for a general proof without relying on the thermal equilibrium. An arbitrary CTP component of the Pauli-Villars regularized one-loop AVV triangle reads

$$
\begin{aligned}
\Delta_{\mu \nu \rho}^{a b c}\left(Q_{1}, Q_{2}\right)= & -\int \frac{d^{4} p}{(2 \pi)^{4}} \operatorname{Tr} \Gamma_{\rho} \gamma_{5} \eta_{c}\left[S\left(p-Q_{2} \mid 0\right) \Gamma_{\nu} \eta_{b} S(p \mid 0) \Gamma_{\mu} \eta_{a} S\left(p+Q_{1} \mid 0\right)+S\left(p-Q_{1} \mid 0\right) \Gamma_{\mu} \eta_{a} S(p \mid 0) \Gamma_{\nu} \eta_{b} S\left(p+Q_{2} \mid 0\right)\right. \\
& \left.-S\left(p-Q_{2} \mid M\right) \Gamma_{\nu} \eta_{b} S(p \mid M) \Gamma_{\mu} \eta_{a} S\left(p+Q_{1} \mid M\right)-S\left(p-Q_{1} \mid M\right) \Gamma_{\mu} \eta_{a} S(p \mid M) \Gamma_{\nu} \eta_{b} S\left(p+Q_{2} \mid M\right)\right],
\end{aligned}
$$

with the trace extending to both spinor and CTP indices. Taking the divergence with respect to the axial vector vertex, we have

$$
\begin{aligned}
i\left(Q_{1}+Q_{2}\right)_{\rho} \Delta_{\rho \mu \nu}^{c a b}\left(Q_{1}, Q_{2}\right)= & -i \int \frac{d^{4} p}{(2 \pi)^{4}}\left[F_{\mu \nu}^{c a b}\left(p-Q_{2}, p\right)-F_{\mu \nu}^{c a b}\left(p, p+Q_{2}\right)+F_{\nu \mu}^{c b a}\left(p-Q_{1}, p\right)-F_{\nu \mu}^{c b a}\left(p, p+Q_{1}\right)\right] \\
& +2 M \int \frac{d^{4} p}{(2 \pi)^{4}} \operatorname{Tr}_{5} \eta_{5}\left[S\left(p-Q_{2} \mid M\right) \Gamma_{\nu} \eta_{b} S(p \mid M) \Gamma_{\mu} \eta_{a} S\left(p+Q_{1} \mid M\right)\right. \\
& \left.+S\left(p-Q_{1} \mid M\right) \Gamma_{\mu} \eta_{a} S(p \mid M) \Gamma_{\nu} \eta_{b} S\left(p+Q_{2} \mid M\right)\right]
\end{aligned}
$$

with $F_{\mu \nu}^{c a b}(p, q) \equiv \operatorname{Tr}\left[\Gamma_{\mu} \gamma_{5} \eta_{c} \eta_{a} S(p \mid 0) \Gamma_{\nu} \eta_{b} S(q \mid 0)-\Gamma_{\mu} \gamma_{5} \eta_{c} \eta_{a} S(p \mid M) \Gamma_{\nu} \eta_{b} S(q \mid M)\right]$, where the identity (42) has been employed. Note that the four terms in the integrand in the first line of (45) are regularized à la Pauli-Villars scheme. Thus it is legitimate to shift the integration momentum and the integral of these terms vanishes. We end up with the contribution from the regulator only, i.e.,

$$
\begin{aligned}
i\left(Q_{1}+Q_{2}\right)_{\rho} \Delta_{\mu \nu \rho}^{a b c}\left(Q_{1}, Q_{2}\right)= & 2 M \int \frac{d^{4} p}{(2 \pi)^{4}} \operatorname{Tr}_{5} \eta_{c}\left[S\left(p-Q_{2} \mid M\right) \Gamma_{\nu} \eta_{b} S(p \mid M) \Gamma_{\mu} \eta_{a} S\left(p+Q_{1} \mid M\right)\right. \\
& \left.+S\left(p-Q_{1} \mid M\right) \Gamma_{\mu} \eta_{a} S(p \mid M) \Gamma_{\nu} \eta_{b} S\left(p+Q_{2} \mid M\right)\right] .
\end{aligned}
$$

In the limit $M \rightarrow \infty$, all Fermi distribution functions embedded in CTP propagators in (39) can be dropped. Among the eight CTP components $(111,112,121,211,122,212,221,222)$, the 111 component corresponds to the Feynman diagram at zero temperature and generates the standard Adler-Bardeen anomaly. Because of the antitime ordering (opposite sense of the Wick rotation), the contribution of the 222 component takes the negative value of the 111 component. As for the other six CTP components, they all carry the combination of the form $S_{12}(p \mid M) S_{21}(p+q \mid M)$ with $q$ the external momentum entering the vertex $S_{12}$ and $S_{21}$. It follows from (39) that in the limit $M \rightarrow \infty$ the energy integration of these components

$$
\int_{\infty}^{\infty} d p_{0} \delta\left(p_{0}+E_{\mathbf{p}}\right) \delta\left(p_{0}+q_{0}-E_{\mathbf{p}+\mathbf{q}}\right)(\ldots)=\delta\left(E_{\mathbf{p}}+E_{\mathbf{p}+\mathbf{q}}-q_{0}\right)(\ldots) \rightarrow 0 .
$$

Consequently, only the 111 and 222 CTP components of the AVV triangle divergence are left over and can be coded in a twopoint CTP vertex

$$
\left(Q_{1}+Q_{2}\right)_{\rho} \Delta_{\mu \nu \rho}^{a b c}\left(Q_{1}, Q_{2}\right)= \begin{cases}-i \frac{e^{2}}{4 \pi^{2}} \epsilon_{\mu \nu \rho \lambda} Q_{1 \rho} Q_{2 \lambda}, & a=b=c=1 \\ i \frac{e^{2}}{4 \pi^{2}} \epsilon_{\mu \nu \rho \lambda} Q_{1 \rho} Q_{2 \lambda}, & a=b=c=2 \\ 0, & \text { otherwise }\end{cases}
$$


Inserting (48) with $Q_{1}=k_{1}, Q_{2}=-k_{2}$, and $\mathbf{k}_{1}=\mathbf{k}_{2}$ into the 4-divergence of the CTP amplitude of the diagram Fig. 1, we obtain, to the leading order in $Q_{0}=k_{10}-k_{20}$, the CTP counterpart of (13)

$$
\begin{aligned}
\Lambda_{\rho \lambda}(q)= & -Q_{0} \frac{e^{6}}{2 \pi^{2}} \epsilon_{m n l} \sum_{a b c d}(-)^{c-1} \int \frac{d^{4} k}{(2 \pi)^{4}} k_{l} D^{a c}(k) \\
& \times D^{b c}(k) \Gamma_{m n \rho \lambda}^{a b 1 d}(q, q ; k), \\
= & Q_{0} L_{\rho \lambda}(q)
\end{aligned}
$$

with the CTP photon propagator $D^{a b}(k) \delta_{\mu \nu}$ in the Feynman gauge and the CTP four-photon box amplitude $\Gamma_{m n i j}^{a b 1 d}(q, q ; k)$ as $Q_{0} \rightarrow 0$. The coefficient of $Q_{0}$,

$$
\begin{aligned}
L_{\rho \lambda}(q)= & -\frac{e^{6}}{2 \pi^{2}} \epsilon_{m n l} \sum_{a b c d}(-)^{c-1} \int \frac{d^{4} k}{(2 \pi)^{4}} k_{l} D^{a c}(k) \\
& \times D^{b c}(k) \Gamma_{m n \rho \lambda}^{a b 1 d}(q, q ; k),
\end{aligned}
$$

with $(\rho, \lambda)=(i, j)$ is the 3-loop contribution to the kernel $K_{i j}(q)$ of the chiral magnetic current. As is discussed above, $L_{i j}(q)$ in response to a static magnetic field can be evaluated à la Matsubara formulation. We have
$L_{i j}(\mathbf{q})=-\frac{e^{6}}{2 \pi^{2}} \epsilon_{m n l} T \sum_{n} \int \frac{d^{3} \mathbf{k}}{(2 \pi)^{3}} k_{l} \frac{1}{\left(k^{2}\right)^{2}} \Gamma_{m n i j}^{(M)}(q, q ; k)$,

where $k=(\mathbf{k},-2 n \pi T)$ with $n$ an integer, $q=(\mathbf{q}, 0)$, and the Matsubara amplitude of the four-photon box diagram reads

$$
\begin{aligned}
\Gamma_{m n i j}^{M}(q, q ; k)= & \mathrm{I}^{(M)}+\mathrm{II}^{(M)}+\mathrm{III}^{(M)}+\mathrm{IV}^{(M)} \\
& +\mathrm{V}^{(M)}+\mathrm{VI}^{(M)}
\end{aligned}
$$

with $\mathrm{I}^{(M)}, \ldots \mathrm{VI}^{(M)}$ obtained by the following replacement in $\mathrm{I}, \ldots, \mathrm{VI}$ of $(15)$,

$$
p \rightarrow(\mathbf{p},-(2 n+1) \pi T), \quad \int \frac{d^{4} k}{(2 \pi)^{4}} \rightarrow T \sum_{n} \int \frac{d^{3} \mathbf{k}}{(2 \pi)^{3}} .
$$

All derivative formulas at zero temperature can be generalized to the Matsubara amplitude as long as the derivatives are with respect to the spatial components $\mathbf{q}$ or $\mathbf{p}$. In particular, we have

$$
\begin{aligned}
\epsilon_{m n l} \Gamma_{n m i j}^{(M)}(0,0 ; k) & =i T \sum \int \frac{d^{3} \mathbf{p}}{(2 \pi)^{3}} \frac{\partial}{\partial p_{j}} \epsilon_{m n l} \operatorname{tr} \gamma_{m}\left(\frac{1}{\not p-\not l_{1}} \gamma_{n} \frac{1}{\not p} \gamma_{i} \frac{1}{\not p}+\frac{1}{\not p} \gamma_{i} \frac{1}{\not p} \gamma_{n} \frac{1}{\not p+\not k}\right), \\
& =i T \sum \int \frac{d^{3} \mathbf{p}}{(2 \pi)^{3}} \frac{\partial}{\partial p_{i}} \epsilon_{m n l} \operatorname{tr} \gamma_{m}\left(\frac{1}{\not \not-\not l_{1}} \gamma_{n} \frac{1}{\not p} \gamma_{j} \frac{1}{\not \not p}+\frac{1}{\not p} \gamma_{j} \frac{1}{\not p} \gamma_{n} \frac{1}{\not p+\not k}\right),
\end{aligned}
$$

and the coefficient of the linear term in $\mathbf{q}$ of $\Gamma_{m n i j}^{(M)}(q, q, k)$ reads

$$
\begin{aligned}
\left.\frac{\partial}{\partial q_{h}} \Gamma_{m n i j}^{(M)}(q, q ; k)\right|_{q=0} & =\left.\frac{\partial}{\partial q_{1 h}} \Gamma_{m n i j}^{(M)}\left(q_{1}, 0 ; k\right)\right|_{q_{1}=0}+\left.\frac{\partial}{\partial q_{2 h}} \Gamma_{m n i j}^{(M)}\left(0, q_{2} ; k\right)\right|_{q_{2}=0}, \\
& =-T \sum \int \frac{d^{3} \mathbf{p}}{(2 \pi)^{3}}\left[\frac{\partial}{\partial p_{j}} \operatorname{tr} \gamma_{m}\left(\frac{1}{\not \supset-\not h} \gamma_{n} \frac{1}{\not p} \gamma_{h} \frac{1}{\not p} \gamma_{i} \frac{1}{\not p}-\frac{1}{\not p} \gamma_{i} \frac{1}{\not p} \gamma_{h} \frac{1}{\not \not p} \gamma_{n} \frac{1}{\not p+\not k}\right)-(i \leftrightarrow j)\right] .
\end{aligned}
$$

Unlike the zero temperature case, the denominator of a Matsubara Dirac propagator never vanishes because of the nonzero fermionic Matsubara energy. Consequently, the Stokes theorem can be applied safely to the integration of the loop momentum, which yields

$$
\begin{aligned}
\Gamma_{m n i j}^{(M)}(0,0 ; k) & =0, \\
\left.\frac{\partial}{\partial q_{h}} \Gamma_{m n i j}^{(M)}(q, q ; k)\right|_{q=0} & =0,
\end{aligned}
$$

because the integrand vanishes fast enough as the momentum tends to infinity. Therefore, the radiative correction that contributes to the chiral magnetic current at zero temperature no longer contributes when the temperature $T \gg q$.

\section{The order $\lim _{\mathrm{Q} \rightarrow 0} \lim _{Q_{0} \rightarrow 0}$ :}

The first limit $Q_{0} \rightarrow 0$ renders the axial chemical potential static. Given the momentum carried by the external vector potential, $q_{1}=\left(\mathbf{q}_{1}, \omega\right)$, and that carried by the current, $q_{2}=\left(\mathbf{q}_{2}, \omega\right)$, the momentum flowing in through the axial chemical potential is $\left(\mathbf{q}_{2}-\mathbf{q}_{1}, 0\right)$. Because of the nonzero spatial momentum at the temporal component of the axial vector vertex, the top triangle is no longer tied to the axial anomaly and its dependence on the internal momentum $k$ becomes more complicated than (48). 
In addition, its contribution may not be equivalent to a bare CTP vertex like in the preceding subsection (diagonal with respect to CTP indices). It follows from the rotation symmetry that the only nonzero component of $\Delta_{\mu \nu 4}^{a b c}(k, k)$ reads

$$
\Delta_{i j 4}^{a b c}(k, k)=i \frac{e^{2}}{2 \pi^{2}} \Delta^{a b c}\left(\mathbf{k}^{2}, k_{0}\right) \epsilon_{i j l} k_{l},
$$

with $\Delta^{a b c}\left(\mathbf{k}^{2}, k_{0}\right)$ a scalar form factor, and we have

$$
\begin{aligned}
L_{\rho \lambda}(q)= & -i \frac{e^{6}}{2 \pi^{2}} \epsilon_{m n l} \sum_{a b c d} \int \frac{d^{4} k}{(2 \pi)^{4}} \Delta^{a b c}\left(\mathbf{k}^{2}, k_{0}\right) \\
& \times D^{a a^{\prime}}(k) D^{b b^{\prime}}(k) \Gamma_{m n \rho \lambda}^{a b 1 d}(q, q ; k)
\end{aligned}
$$

in contrast to (50) above. Nevertheless, for the standard setup underlying (5), all external momenta are static, and the retarded CTP Green's function reduces to the Matsubara one, which shares the same four-photon box as in the preceding subsection. Following the argument there, in the limit $\mathbf{q}_{2} \rightarrow \mathbf{q}_{\mathbf{1}} \equiv \mathbf{q}$, the term of the four-photon box that is linear in $\mathbf{q}$ vanishes because of (56) and the threeloop diagram in Fig. 1 does not contribute to the chiral magnetic current (5) in this order of limits.

A cautious reader may wonder if the integration over the two internal photon lines will bring an inverse power in $|\mathbf{q}|$. This possibility can be ruled out for QED and for QCD up to $O\left(g^{4}\right)$. Though the infrared behavior of the photon or gluon propagator becomes worse in the Matsubara formulation when its energy vanishes, which is the underlying mechanism of the Linde's problem of a pure Yang-Mills gas [26], it does not cause infrared divergence here. By turning around the argument leading to (56), we can prove that

$$
\begin{aligned}
\Gamma_{m n i j}^{(M)}(q, q ; 0) & =0, \\
\left.\frac{\partial}{\partial k_{h}} \Gamma_{m n i j}^{(M)}(q, q ; k)\right|_{k=0} & =0 .
\end{aligned}
$$

Combining (56) and (59), we find that

$$
\Gamma_{m n i j}^{(M)}=O\left(\mathbf{k}^{2} \mathbf{q}^{2}\right)
$$

at $q_{0}=k_{0}=0$ for $|\mathbf{q}| \ll T$ and $|\mathbf{k}| \ll T$. Consequently, the integration of the spatial momentum of the two internal photon lines or gluon lines in Fig. 1 at zero Matsubara energy is infrared safe, and our statement in the preceding paragraph is not modified. When the fourphoton boxes are chained as in Fig. 2, the estimation (60) with q not restricted to the external photon momentum ensures the infrared convergence of the momentum integrals of all internal photon lines as $\mathbf{q} \rightarrow 0$ if none of them are temporal. On the other hand, a photon line not attached to the anomalous triangle can be temporal and (60) does not apply. In this case, the Debye mass serves the required infrared cutoff [27]. Applying (56) to the four-photon box at the bottom of Fig. 2, we thereby rule out the radiation corrections to CME from the chain of the four-photon box in Fig. 2 in both orders of the constant axial chemical potential limit. For QCD, there is no simple systematics like Fig. 2 to higher orders because of the selfcoupling of gluons. Our statement on the absence of the radiative correction is limited to the three-loop diagram Fig. 1 with the two internal photon lines replaced by two gluon lines, and this is of the order $O\left(g^{4}\right)$ in Yang-Mills coupling.

While the absence of the radiative correction in the Matsubara formulation follows from the four-dimensional generalization of the Coleman-Hill theorem [28] for the three-dimensional QED, the key step here is to recognize that the real time amplitude, where the infrared behavior is difficult to track, becomes the Matsubara amplitude after setting all external momenta static, and the Coleman-Hill theorem can be applied to the four-photon box then because of the benign infrared behavior of the Dirac propagator. The subsequent discussions, after Eq. (51), provide a concrete implementation of the theorem. See the Appendix of [28] for an alternative proof of (60). Though the radiative correction brought about by the diagram of Fig. 1 does not contribute to the chiral magnetic current in a static and uniform magnetic field, it does contribute to the current in a static but inhomogeneous magnetic field through higher powers in $|\mathbf{q}|$. The difference between the two orders of the limit (in subsections III A and III B) as a reminiscence of the real time Green's function remains there.

The kinematic region for the absence of the radiative correction from Figs. 1 and 2 to CME, $|\mathbf{q}| \ll T$, does not include the zero temperature point. As the temperature is lowered at a fixed $|\mathbf{q}|$, the higher powers of $|\mathbf{q}|$ become more important, and the radiative corrections to CME are gradually built up until $T \ll|\mathbf{q}|$, then the radiative correction discussed in Sec. II emerges and the difference between the two orders of limits diminishes.

\section{SUMMARY AND CONCLUSIONS}

In this work, we analyzed the impact of a higher-order correction to the matrix element of the axial anomaly in massless QED. Through the interplay between the vector Ward identity and the infrared singularity with massless fermions, we are able to rederive, in a much simpler way, the known corrections from the three-loop diagram in Fig. 1 at zero temperature. This type of radiative correction does not invalidate the Adler-Bardeen theorem since the kinematic point required by the theorem cannot be reached for a massless fermion field, but it does contribute to the chiral magnetic current in the massless limit at zero temperature. 
Then we move on to the case of a nonzero temperature where the limit of zero energy momentum along an external line becomes subtle. We consider two orders of zero energy-momentum limit pertaining to the axial chemical potential: (1) starting with a homogeneous axial chemical potential where its time dependence is switched off later and (2) starting with a static axial chemical potential where its inhomogeneity is removed later. Though the real time formulation has to be employed to carry out the zero energy limit, the Matsubara formulation suffices afterward for a static magnetic field, where the infrared singularity of the massless Dirac propagator at zero temperature disappears and the vector Ward identity of the four-photon box can be applied without hurdles. Consequently, the three-loop diagram does not contribute a radiative correction to the chiral magnetic current in either order of limit. The same conclusion holds for the higher-order diagrams with repeated photon-photon scattering amplitudes in accordance with the Coleman-Hill theorem, and the one-loop results (8) and (9) remain intact for the diagrams in Fig. 2. The absence of the radiative correction also applies to the three-loop diagram with the two internal photon lines replaced by two gluon lines. Not addressed in this paper is the case with a time-dependent magnetic field, which cannot be evaluated with the Matsubara formulation. One has to track the infrared behavior of the full-fledged real time formulation throughout. Denoting the kernel for the response of the electric current to the magnetic field by $K_{i j}^{(s)}(q)$ with $s=A, B$ corresponding to the two orders of limits discussed in subsections III A and III B, our result of the $\mathrm{CME}$ with massless fermions can be summarized in the following:

$$
K_{i j}^{(s)}(\mathbf{q})=i \frac{e^{2}}{2 \pi^{2}} F_{s}\left(\frac{|\mathbf{q}|}{T}\right) \epsilon_{i k j} q_{k} .
$$

We find that $F_{A}(|\mathbf{q}| / T) \rightarrow 1$ and $F_{B}(|\mathbf{q}| / T) \rightarrow 0$ as $|\mathbf{q}| / T \rightarrow 0$ for all QED diagrams in Fig. 2 and up to $O\left(g^{4}\right)$ QCD corrections. In the low-temperature limit, $|\mathbf{q}| / T \rightarrow \infty$

$$
F_{s}(|\mathbf{q}| / T) \rightarrow 1-\frac{3 e^{4}}{64 \pi^{4}} \log \frac{\Lambda^{2}}{q^{2}},
$$

for the three-loop QED diagrams of Fig. 1, and

$$
F_{s}(|\mathbf{q}| / T) \rightarrow 1-\frac{3 g^{4}}{32 \pi^{4}} \log \frac{\Lambda^{2}}{q^{2}} .
$$

if the two internal photon lines in Fig. 1 are replaced by gluon lines.

It is interesting to note that the difference between the operator anomaly and its matrix element at zero temperature is carried over to the nonzero temperature case. At zero temperature, the form of the axial anomaly at the operator level does not require a special kinematic point but its matrix element does. So is the case at a nonzero temperature, the chiral magnetic current (5) implied by the one-loop axial anomaly (48), holds for an arbitrary spacetime-dependent magnetic field. Its validity can be extended beyond thermal equilibrium since the identity (42) does not rely on the specific form of the CTP propagator (39). With the final state photon-photon scattering, however, the validity of (5) is limited to the low external momenta for massive fermions at zero temperature and is limited to a static and homogeneous magnetic field at a nonzero temperature. The temperature here plays a similar role of infrared cutoff as the fermion mass at zero temperature.

While our analysis supports the absence of radiative correction to the chiral magnetic current under a static and homogeneous magnetic field in massless QED at a nonzero temperature. The QCD corrections, however, are much more difficult to assess in general. Though we are able to rule out the radiative correction from the diagram with the two internal photon lines replaced by two gluon lines, the higher-order QCD diagram cannot be decomposed as simply as Fig. 2 because of the gluon's self-coupling. Within the framework of Matsubara formulation, the potential infrared singularity comes from the terms with zero Matsubara energies along all internal gluon and photon lines [26]. A power-counting argument analogous to that in [29] yields a contribution of the order

$$
\left(\frac{|\mathbf{q}|}{T}\right)^{\frac{1}{2} V_{\gamma}+\frac{1}{2} V_{q}-\frac{1}{2} V_{3}-V_{4}+1}
$$

to $F_{s}(|\mathbf{q}| / T)$ of (61), where $V_{\gamma}, V_{q}, V_{3}$, and $V_{4}$ are the numbers of the quark-photon, quark-gluon, 3-gluon, and 4-gluon vertices. Such an infrared catastrophe with increasing numbers of gluon self-coupling vertices may be regulated by a nonperturbative chromomagnetic mass. A full nonperturbative calculation, say lattice simulation, is yet required to assess the robustness of the classical form of the chiral magnetic current (5) in the presence of the gluon dynamics.

In noncentral heavy ion collisions, the magnetic field created therein is both transient and inhomogeneous. The spatial distribution of the magnetic field is dominated in the plane perpendicular to the reaction plane and the spatial size, according to the simulations [30-32], is about $5 \mathrm{fm}$ in collisions at center-of-mass energy $\sqrt{s}=200 \mathrm{GeV}$. Therefore, the spatial momentum carried by the external vector potential $|\mathbf{q}|$ is roughly about $40 \mathrm{MeV}$, which is smaller than the temperature $T \sim 200 \mathrm{MeV}$ reached in the collision. But the lifetime is about $1 \sim 2 \mathrm{fm}$, making $|\mathbf{q}| \ll$ $q_{0}$ outside the kinetic region $|\mathbf{q}|>q_{0}$ covered here. As discussed in Sec. III, the real time formulation has to be employed throughout the analysis to tackle the radiative corrections for $|\mathbf{q}| \ll q_{0}$, and $|\mathbf{q}| \ll T$, in which case the 
infrared behavior is more convoluted to track. This is currently being explored, and the result will be reported in the near future.

The radiative corrections to the anomalous transport coefficients are also discussed in the literature in the context of the chiral vortical effect $[33,34]$ and the chiral separation effect [35]. The mechanism of the radiative correction in $[33,34]$ is similar to the final state interaction discussed in this work, where the two photons coming from the anomalous triangle combine into a graviton, but the correction is nonvanishing only at a nonzero temperature. The radiative corrections in [35] come from the component of the two-loop VA two-point function that is not dictated by the axial anomaly.

$$
\begin{aligned}
\Lambda_{\rho \lambda}\left(q_{1}, q_{2}\right)= & -i \frac{e^{6}}{2 \pi^{2}} \epsilon_{\alpha \mu \beta \nu} \int \frac{d^{4} p}{(2 \pi)^{4}} \int \frac{d^{4} k_{1}}{(2 \pi)^{4}} Q_{\alpha} k_{1 \beta} \frac{1}{\left(k_{1}^{2}\right)^{2}} \\
& \times \operatorname{tr}\left[2 \frac{\not p}{p^{2}} \gamma_{\mu} \frac{\not p-\not k_{1}}{\left(p-k_{1}\right)^{2}} \gamma_{\nu} \frac{\not p}{p^{2}} \gamma_{\lambda} \frac{\not p+\not q_{1}}{\left(p+q_{1}\right)^{2}} \gamma_{\rho}+\frac{\not p}{p^{2}} \gamma_{\mu} \frac{\not p-\not k_{1}}{\left(p-k_{1}\right)^{2}} \gamma_{\rho} \frac{\not p-\not k_{1}+\not q_{2}}{\left(p-k_{1}+q_{2}\right)^{2}} \gamma_{\nu} \frac{\not p-\not q_{2}}{\left(p-q_{2}\right)^{2}} \gamma_{\lambda}\right] \\
& +\left(\rho \leftrightarrow \lambda, q_{1} \leftrightarrow q_{2}\right)+\mathcal{O}\left(Q^{2}\right) .
\end{aligned}
$$

Here, we explicitly present the permutation factor connecting the photon box to the triangle diagrams. Note that the divergence of AVV triangle diagram can be regarded as a point and represented by $e^{2} /\left(2 \pi^{2}\right) \epsilon_{\alpha \mu \beta \nu} Q_{\alpha} k_{1 \beta}$, which enables us to ignore completely the $Q$ dependence in the photon box and the internal two photon lines in the leading order of $Q$. In the following calculations, we only aim to keep the UV divergent part and will ignore the finite terms entirely. For this purpose, it is in great advantage to carry out firstly the integral of the photon-box loop momentum $p$ and then that of the internal photon momentum $k_{1}$, because the former integral of the momentum $p$ turns out to be finite.

It can be shown by simple power counting that the potentially UV divergent part in the integral of $p$ reads

$\epsilon_{\alpha \mu \beta \nu} \int \frac{d^{4} p}{(2 \pi)^{4}} \frac{1}{\left(p^{2}\right)^{4}} \operatorname{tr}\left[\gamma_{\lambda} \not \not \gamma_{\mu} \not \not \gamma_{\rho} \not \not \gamma_{\nu} \not p+\gamma_{\rho} \not \not \gamma_{\mu} \not \not \gamma_{\nu} \not \not \gamma_{\lambda} \not p\right]$,

But this integral is vanishing due to the Levi-Civita tensor $\epsilon_{\alpha \sigma \beta \rho}$ that can be shown as follows. Symmetry allows one to replace

\section{ACKNOWLEDGMENTS}

We are grateful to D. Kharzeev for bringing our attention to the work [19] and the difference between the operator anomaly and its matrix elements. This work is, in part, supported by the Ministry of Science and Technology of China (MSTC) under the 973 Project No. 2015CB856904 (4) and by NSFC under Grants No. 11735007, No. 11890711, and No. 11521064.

The CME current corresponds to the static limit $Q=$ $\left(q_{2}-q_{1}\right) \rightarrow 0$ of the divergence of the amplitudes of diagrams in Fig. 1. One has

\section{APPENDIX A: A STRAIGHTFORWARD CALCULATION OF THE DIAGRAMS IN FIG. 1}$$
\Gamma
$$

$$
p_{\xi} p_{\sigma} p_{\kappa} p_{\delta} \sim \frac{1}{24}\left(p^{2}\right)^{2}\left(\delta_{\xi \sigma} \delta_{\kappa \delta}+\delta_{\xi \kappa} \delta_{\sigma \delta}+\delta_{\xi \delta} \delta_{\sigma \kappa}\right),
$$

The vanishing of the first term in Eq. (A2) can be shown by noting that

$$
\begin{gathered}
\left(\delta_{\xi \sigma} \delta_{\kappa \delta}+\delta_{\xi \kappa} \delta_{\sigma \delta}+\delta_{\xi \delta} \delta_{\sigma \kappa}\right) \epsilon_{\alpha \mu \beta \nu} \\
\times \operatorname{tr}\left(\gamma_{\lambda} \gamma_{\xi} \gamma_{\mu} \gamma_{\sigma} \gamma_{\rho} \gamma_{\kappa} \gamma_{\nu} \gamma_{\delta}\right)=0,
\end{gathered}
$$

which renders the photon-box diagram (b) in Fig. 1 finite. Likewise, the same photon-box diagram $(b)$ in Fig. 1 with two final photon states interchanged is also finite. The second term in Eq. (A2) is, however, nonzero

$$
\begin{aligned}
& \left(\delta_{\xi \sigma} \delta_{\kappa \delta}+\delta_{\xi \kappa} \delta_{\sigma \delta}+\delta_{\xi \delta} \delta_{\sigma \kappa}\right) \epsilon_{\alpha \mu \beta \nu} \\
& \quad \times \operatorname{tr}\left(\gamma_{\rho} \gamma_{\xi} \gamma_{\mu} \gamma_{\sigma} \gamma_{\nu} \gamma_{\kappa} \gamma_{\lambda} \gamma_{\delta}\right)=96 \epsilon_{\alpha \rho \beta \lambda} .
\end{aligned}
$$

But the sum of (A5) and the one with two final photon states interchanged is zero due to the antisymmetry of the Levi-Civita tensor $\epsilon_{\alpha \rho \beta \lambda}$, which renders the photon-box diagram (a) finite.

Performing the finite integral of momentum $p$ and keeping only the UV divergent terms upon the integral of $k_{1}$, one has

$$
\begin{aligned}
\Lambda_{\rho \lambda}\left(q_{1}, q_{2}\right)= & \frac{e^{6}}{8 \pi^{4}} Q_{\alpha} q_{1 \beta} \epsilon_{\alpha \rho \beta \lambda} \int^{\Lambda} \frac{d^{4} k_{1}}{(2 \pi)^{4}} \frac{1}{\left(k_{1}^{2}\right)^{2}} \int_{0}^{1} d y \int_{0}^{1-y} d w(1-y-w) \\
& \times\left[\frac{12 y w^{2}-22 y w-6 w^{2}+9 w+9 y-3}{w(w-1)^{2}}+\frac{1}{(y+w)(y+w-1)}\right]+\left(\lambda \leftrightarrow \rho, q_{1} \leftrightarrow q_{2}\right) .
\end{aligned}
$$


with $\Lambda$ a UV cutoff. We note that the result is logarithmically divergent. Carrying out the integral of Feynman parameters, one obtains

$$
\begin{aligned}
\Lambda_{\rho \lambda}\left(q_{1}, q_{2}\right) & =\frac{-3 e^{6}}{8 \pi^{4}} Q_{\alpha} q_{1 \beta} \epsilon_{\alpha \rho \beta \lambda} \int^{\Lambda} \frac{d^{4} k_{1}}{(2 \pi)^{4}} \frac{1}{\left(k_{1}^{2}\right)^{2}}, \\
& =i \frac{3 e^{6}}{128 \pi^{6}} Q_{\alpha} q_{1 \beta} \epsilon_{\alpha \rho \beta \lambda} \ln \frac{\Lambda^{2}}{k^{2}}
\end{aligned}
$$

with $k$ an infrared cutoff depending on the external momenta.

\section{APPENDIX B: THE WARD IDENTITIES OF THE BOX DIAGRAMS WITH FOUR PHOTONS}

Using the identity

$$
\frac{1}{\not p+\not q} \not \frac{1}{\not p}=\frac{1}{\not p}-\frac{1}{\not p+\not q},
$$

we find that

$$
\begin{aligned}
-i q_{2 \lambda} \Gamma_{\mu \nu \rho \lambda}\left(q_{1}, q_{2} ; k_{1}\right)= & \int \frac{d^{4} p}{(2 \pi)^{4}}\left[G_{\mu \nu \rho}\left(p+q_{2} \mid q_{1}, q_{2} ; k_{1}\right)\right. \\
& \left.-G_{\mu \nu \rho}\left(p \mid q_{1}, q_{2} ; k_{1}\right)\right], \quad \text { (B2) }
\end{aligned}
$$

where

$$
\begin{aligned}
G_{\mu \nu \rho}\left(p \mid q_{1}, q_{2} ; k_{1}\right)= & \operatorname{tr} \gamma_{\mu}\left(\frac{1}{\not p-\not k_{1}} \gamma_{\nu} \frac{1}{\not p-\not q_{2}+\not q_{1}} \gamma_{\rho} \frac{1}{\not p-\not q_{2}}\right. \\
& \left.+\frac{1}{\not p} \gamma_{\rho} \frac{1}{\not p-\not \not_{1}} \gamma_{\nu} \frac{1}{\not p-\not q_{2}+\not l_{1}}\right),
\end{aligned}
$$

and

$$
\begin{aligned}
-i q_{1 \rho} \Gamma_{\mu \nu \rho \lambda}\left(q_{1}, q_{2} ; k_{1}\right)= & \int \frac{d^{4} p}{(2 \pi)^{4}}\left[H_{\mu \nu \lambda}\left(p+k_{1} \mid q_{1}, q_{2} ; k_{1}\right)\right. \\
& -H_{\mu \nu \lambda}\left(p \mid q_{1}, q_{2} ; k_{1}\right) \\
& +I_{\mu \nu \lambda}\left(p-k_{1}+q_{1} \mid q_{1}, q_{2} ; k_{1}\right) \\
& \left.-I_{\mu \nu \lambda}\left(p \mid q_{1}, q_{2} ; k_{1}\right)\right], \quad \text { (B4) }
\end{aligned}
$$

where

$$
\begin{aligned}
H_{\mu \nu \lambda}\left(p \mid q_{1}, q_{2} ; k_{1}\right)= & \operatorname{tr} \gamma_{\mu}\left(\frac{1}{\not p-\not \not_{1}} \gamma_{\nu} \frac{1}{\not p+\not q_{2}} \gamma_{\lambda} \frac{1}{\not p}\right) \\
& +\operatorname{tr} \gamma_{\mu}\left(\frac{1}{\not p-\not k_{1}} \gamma_{\lambda} \frac{1}{\not p-\not k_{1}+\not \not_{2}} \gamma_{\nu} \frac{1}{\not p}\right),
\end{aligned}
$$

and

$$
\begin{aligned}
I_{\mu \nu \lambda}\left(p \mid q_{1}, q_{2} ; k_{1}\right)= & \operatorname{tr} \gamma_{\mu}\left(\frac{1}{\not p-\not \not_{1}} \gamma_{\nu} \frac{1}{\not p+\not k_{1}-\not q_{2}} \gamma_{\lambda} \frac{1}{\not p+\not k_{1}}\right) \\
& +\operatorname{tr} \gamma_{\mu}\left(\frac{1}{\not p-\not q_{1}} \gamma_{\lambda} \frac{1}{\not p-\not q_{1}-\not q_{2}} \gamma_{\nu} \frac{1}{\not p+\not k_{1}}\right) .
\end{aligned}
$$

Both $G_{\mu \nu \rho}\left(p \mid q_{1}, q_{2} ; k_{1}\right)$ and $H_{\mu \nu \lambda}\left(p \mid q_{1}, q_{2} ; k_{1}\right)$ give rise to linearly divergent integrals by power counting and a regulator is required to justify the shift of the integration momentum $p$ and thereby the Ward identity. On the other hand, the leading terms in $p$ are canceled in the combinations $\epsilon_{\mu \nu \alpha \lambda} G_{\mu \nu \rho}\left(p \mid q_{1}, q_{2} ; k_{1}\right)$ and $\epsilon_{\mu \nu \alpha \beta} H_{\mu \nu \rho}\left(p \mid q_{1}, q_{2} ; k_{1}\right)$ and the integration becomes logarithmic divergence by power counting. Consequently, the Ward identities

$$
\begin{aligned}
& -i q_{2 \lambda} \epsilon_{\mu \nu \alpha \beta} \Gamma_{\mu \nu \rho \lambda}\left(q_{1}, q_{2} ; k_{1}\right)=0, \\
& -i q_{1 \rho} \epsilon_{\mu \nu \alpha \beta} \Gamma_{\mu \nu \rho \lambda}\left(q_{1}, q_{2} ; k_{1}\right)=0,
\end{aligned}
$$

without the need of regulators.

\section{APPENDIX C: A NON-PERTURBATIVE PROOF OF THE RELATION BETWEEN THE REAL TIME AND MATSUBARA FORMULATIONS OF A THREE-POINT GREEN'S FUNCTION}

In this Appendix, we discuss the relation between the real time and Matsubara formulations of a three-point Green's function. In terms of the spectral representation, our result applies to all orders of perturbation theory. Let $A\left(t_{1}\right), B\left(t_{2}\right)$, and $C\left(t_{3}\right)$ be field operators at real times $t_{1}, t_{2}$, and $t_{3}$ in the Heisenberg representation with the time evolution

$$
O(t)=e^{i H t} O(0) e^{-i H t},
$$

where $H$ is the Hamiltonian of the system. The different symbols, $A, B$, and $C$, reflect different field operators or the same field operators evaluated at different spatial coordinates. For CME discussed in this work, $A$ and $B$ stand for the electric current density with $B$ attached to the external electromagnetic potential and $C$ represents the axial current density attached to the external axial vector potential. It follows from the CTP formulation that the electric current measured at time $t_{1}$ in response to the external field at $t_{2}$ and $t_{3}$ is given by the three-point retarded Green's function

$G_{R}\left(t, t^{\prime}\right) \equiv G_{111}\left(t, t^{\prime}\right)-G_{121}\left(t, t^{\prime}\right)-G_{112}\left(t, t^{\prime}\right)+G_{122}\left(t, t^{\prime}\right)$,

where $t \equiv t_{1}-t_{2}, t^{\prime} \equiv t_{1}-t_{3}$ and the different CTP components on the rhs are given explicitly by 


$$
\begin{aligned}
& G_{111}\left(t, t^{\prime}\right)=\left\langle T\left(A_{1} B_{2} C_{3}\right)\right\rangle \\
& G_{121}\left(t, t^{\prime}\right)=\left\langle B_{2} T\left(A_{1} C_{3}\right)\right\rangle \\
& G_{112}\left(t, t^{\prime}\right)=\left\langle C_{3} T\left(A_{1} B_{2}\right)\right\rangle \\
& G_{122}\left(t, t^{\prime}\right)=\left\langle\tilde{T}\left(B_{2} C_{3}\right) A_{1}\right\rangle,
\end{aligned}
$$

where $A_{1} \equiv A\left(t_{1}\right), B_{2} \equiv B\left(t_{2}\right), C_{3} \equiv C\left(t_{3}\right)$, and $T(\tilde{T})$ imposes time (antitime) ordering. The expectation value is

$$
\langle O\rangle \equiv \frac{\operatorname{Tr} e^{-\beta H} O}{\operatorname{Tr} e^{-\beta H}}
$$

with $\beta$ the inverse temperature and $Z \equiv \operatorname{Tr} e^{-\beta H}$. Expanding the time and antitime ordering product, we have

$$
\begin{aligned}
G_{R}\left(t, t^{\prime}\right)= & \left\langle A_{1} B_{2} C_{3}\right\rangle \theta_{123}+\left\langle B_{2} C_{3} A_{1}\right\rangle \theta_{231}+\left\langle C_{3} A_{1} B_{2}\right\rangle \theta_{312}+\left\langle B_{2} A_{1} C_{3}\right\rangle \theta_{213}+\left\langle A_{1} C_{3} B_{2}\right\rangle \theta_{132} \\
& +\left\langle C_{3} B_{2} A_{1}\right\rangle \theta_{321}-\left\langle B_{2} A_{1} C_{3}\right\rangle \theta_{13}-\left\langle B_{2} C_{3} A_{1}\right\rangle \theta_{31}-\left\langle C_{3} A_{1} B_{2}\right\rangle \theta_{12}-\left\langle C_{3} B_{2} A_{1}\right\rangle \theta_{21} \\
& +\left\langle C_{3} B_{2} A_{1}\right\rangle \theta_{23}+\left\langle B_{2} C_{3} A_{1}\right\rangle \theta_{32}
\end{aligned}
$$

where $\theta_{a b} \equiv \theta\left(t_{a}-t_{b}\right)$ and $\theta_{a b c} \equiv \theta\left(t_{a}-t_{b}\right) \theta\left(t_{b}-t_{c}\right)=\theta_{a b} \theta_{b c}$ The spectral representation amounts to inserting the complete set of eigenstates of $H, H|N\rangle=E_{N}|N\rangle$, with

$$
\langle N \mid M\rangle=\delta_{N M} 1=\sum_{N}|N\rangle\langle N|,
$$

for instance,

$$
\begin{aligned}
\left\langle A_{1} B_{2} C_{3}\right\rangle & =\frac{1}{Z} \sum_{N, M, L} e^{-\beta E_{N}}\left\langle N\left|A\left(t_{1}\right)\right| M\right\rangle\left\langle M\left|B\left(t_{2}\right)\right| L\right\rangle\left\langle L\left|C\left(t_{3}\right)\right| N\right\rangle \\
& =\frac{1}{Z} \sum_{N, M, L} e^{-\beta E_{N}}\langle N|A(0)| M\rangle\langle M|B(0)| L\rangle\langle L|C(0)| N\rangle e^{i\left(E_{N}-E_{M}\right) t_{1}+i\left(E_{M}-E_{L}\right) t_{2}+i\left(E_{L}-E_{N}\right) t_{3}} \\
& =\frac{1}{Z} \sum_{N, M, L} e^{-\beta E_{N}}(A B C) e^{i\left(E_{N}-E_{L}\right) t^{\prime}+i\left(E_{L}-E_{M}\right) t} .
\end{aligned}
$$

Applying this procedure to all terms of (C5) and then transforming to the energy representation, we obtain that

$$
\begin{aligned}
\mathcal{G}_{R}\left(\omega, \omega^{\prime}\right) \equiv & \int_{-\infty}^{\infty} d t \int_{-\infty}^{\infty} d t^{\prime} e^{i\left(\omega t+\omega^{\prime} t^{\prime}\right)} G_{R}\left(t, t^{\prime}\right) \\
= & \frac{1}{Z} \sum_{N, M, L}(A B C)\left[\frac{e^{-\beta E_{N}}}{\left(\omega^{\prime}+E_{N}-E_{L}+i 0^{+}\right)\left(\omega+\omega^{\prime}+E_{N}-E_{M}+i 0^{+}\right)}\right. \\
& \left.+\frac{e^{-\beta E_{M}}}{\left(\omega+E_{L}-E_{M}+i 0^{+}\right)\left(\omega+\omega^{\prime}+E_{N}-E_{M}+i 0^{+}\right)}-\frac{e^{-\beta E_{L}}}{\left(\omega^{\prime}+E_{N}-E_{L}+i 0^{+}\right)\left(\omega+E_{L}-E_{M}+i 0^{+}\right)}\right] \\
& -\frac{1}{Z} \sum_{N, M, L}(C B A)\left[\frac{e^{-\beta E_{N}}}{\left(\omega^{\prime}+E_{M}-E_{N}+i 0^{+}\right)\left(\omega+\omega^{\prime}+E_{L}-E_{N}+i 0^{+}\right)}\right. \\
& \left.+\frac{e^{-\beta E_{L}}}{\left(\omega+E_{L}-E_{M}+i 0^{+}\right)\left(\omega+\omega^{\prime}+E_{L}-E_{N}+i 0^{+}\right)}-\frac{e^{-\beta E_{M}}}{\left(\omega^{\prime}+E_{M}-E_{N}+i 0^{+}\right)\left(\omega+E_{L}-E_{M}+i 0^{+}\right)}\right]
\end{aligned}
$$

after some cyclic permutations under the trace, where

$$
\begin{aligned}
& (A B C) \equiv\langle N|A(0)| M\rangle\langle M|B(0)| L\rangle\langle L|C(0)| N\rangle \\
& (C B A) \equiv\langle N|C(0)| M\rangle\langle M|B(0)| L\rangle\langle L|A(0)| N\rangle .
\end{aligned}
$$


The poles on the complex- $\omega$ or complex- $\omega^{\prime}$ plane are all located below the real axis as expected. In the static limit, we find that

$$
\begin{aligned}
\mathcal{G}_{R}(0,0)= & -\frac{1}{Z} \sum_{N, M, L}[(A B C)+(B C A)]\left[\frac{e^{-\beta E_{N}}}{\left(E_{N}-E_{L}\right)\left(E_{N}-E_{M}\right)}\right. \\
& \left.+\frac{e^{-\beta E_{M}}}{\left(E_{M}-E_{L}\right)\left(E_{M}-E_{N}\right)}+\frac{e^{-\beta E_{L}}}{\left(E_{L}-E_{M}\right)\left(E_{L}-E_{N}\right)}\right] .
\end{aligned}
$$

There are no singularities when any pair of $E_{N}, E_{M}$, and $E_{L}$ coalesces.

Coming to the Matsubara formulation, the Euclidean time evolution of an operator is generated by

$$
O(\tau)=e^{H \tau} O(0) e^{-H \tau}
$$

with $0 \leq \tau<\beta$. The three-point Green's function corresponding to (C5) reads

$$
\begin{aligned}
G_{M}\left(\tau_{1}, \tau_{2}, \tau_{3}\right) & =\left\langle\mathcal{T}\left[A\left(\tau_{1}\right) B\left(\tau_{2}\right) C\left(\tau_{3}\right)\right]\right\rangle \\
& =\frac{1}{Z}\left[\operatorname{Tr} e^{-\beta H} A\left(\tau_{1}\right) B\left(\tau_{2}\right) C\left(\tau_{3}\right) \theta\left(\tau_{1}-\tau_{2}\right) \theta\left(\tau_{2}-\tau_{3}\right)+5 \text { other permutations }\right],
\end{aligned}
$$

and its Fourier transformation at Matsubara energies $\omega_{1}, \omega_{2}$, and $\omega_{3}$,

$$
\mathcal{G}_{M}\left(\omega_{1}, \omega_{2}, \omega_{3}\right)=\int_{0}^{\beta} d \tau_{1} \int_{0}^{\beta} d \tau_{2} \int_{0}^{\beta} d \tau_{3} e^{i\left(\omega_{1} \tau_{1}+\omega_{2} \tau_{2}+\omega_{3} \tau_{3}\right)} G_{M}\left(\tau_{1}, \tau_{2}, \tau_{3}\right)
$$

In particular, at zero external Matsubara energies,

$$
\mathcal{G}_{M}(0,0,0)=\int_{0}^{\beta} d \tau_{1} \int_{0}^{\beta} d \tau_{2} \int_{0}^{\beta} d \tau_{3} G_{M}\left(\tau_{1}, \tau_{2} \tau_{3}\right)
$$

Inserting the complete set of eigenstates of $H$ as (C7) and carrying out the integral (C14), we end up with

$$
\begin{aligned}
\mathcal{G}_{M}(0,0,0)= & \frac{\beta}{Z} \sum_{N, M, L}[(A B C)+(B C A)]\left[\frac{e^{-\beta E_{N}}}{\left(E_{N}-E_{L}\right)\left(E_{N}-E_{M}\right)}\right. \\
& \left.+\frac{e^{-\beta E_{M}}}{\left(E_{M}-E_{L}\right)\left(E_{M}-E_{N}\right)}+\frac{e^{-\beta E_{L}}}{\left(E_{L}-E_{M}\right)\left(E_{L}-E_{N}\right)}\right] .
\end{aligned}
$$

Comparing with (C15), we find that

$$
\mathcal{G}_{M}(0,0,0)=-\beta \mathcal{G}_{R}(0,0) .
$$

\section{APPENDIX D: A GENERAL PROOF OF IDENTITY (42)}

The identities underlying the vector and axial vector (anomalous) Ward identity,

$$
\begin{aligned}
S_{F}(p+q) \not S_{F}(p)= & i\left[S_{F}(p)-S_{F}(p+q)\right], \\
S_{F}(p+q) \phi q \gamma_{5} S_{F}(p)= & i\left[\gamma_{5} S_{F}(p)+S_{F}(p+q) \gamma_{5}\right] \\
& +2 m S_{F}(p+q) \gamma_{5} S_{F}(p),
\end{aligned}
$$

with $S_{F}(p)$ the free Dirac propagator in Feynman diagrams can be readily generalized to the CTP formulation. Consider the integral

$$
I_{V} \equiv \int_{\mathcal{C}} d^{4} y \frac{\partial \theta}{\partial y_{\mu}} S(x-y) \gamma_{\mu} S\left(y-x^{\prime}\right),
$$

where $S(x-y) \equiv\langle\psi(x) \bar{\psi}(y)\rangle$ with $\langle\ldots\rangle$ the average with respect to the CTP path integral with arbitrary density operator and $\theta(x)$ is an arbitrary function. The time components of all coordinates are along the closed path of the CTP formulation with

$$
\int_{\mathcal{C}} d^{4} y(\ldots) \equiv \int d^{3} \mathbf{y}\left(\int_{-\infty}^{\infty}+\int_{\infty}^{-\infty}\right) d t(\ldots)
$$

By definition,

$$
-\left(\gamma_{\mu} \frac{\partial}{\partial x_{\mu}}+m\right) S(x-y)=i \delta^{4}(x-y)
$$

and 


$$
S(x-y)\left(\overleftarrow{\gamma_{\mu} \frac{\partial}{\partial y_{\mu}}}-m\right)=i \delta^{4}(x-y)
$$

Integrating by part, we find that

$$
I_{V}=-i\left[\theta(x)-\theta\left(x^{\prime}\right)\right] S\left(x-x^{\prime}\right),
$$

with $x$ and $x^{\prime}$ here also containing the information on time branches. Labeling different branches of the closed time path by different CTP indices, $S(x-y)$ becomes a $2 \times 2$ matrix with respect to CTP indices, (D6) becomes

$$
\begin{aligned}
\int_{\mathcal{C}} d^{4} y \frac{\partial \theta_{c}}{\partial y_{\mu}} S_{a c}(x-y) \gamma_{\mu} S_{c b}\left(y-x^{\prime}\right)= & \theta_{c}(x) S_{c b}\left(x-x^{\prime}\right) \\
& -S_{a c}\left(x-x^{\prime}\right) \theta_{c}\left(x^{\prime}\right),
\end{aligned}
$$

or

$$
\begin{aligned}
& \int_{\mathcal{C}} d^{4} y \frac{\partial \theta}{\partial y_{\mu}} S(x-y) \eta_{c} \gamma_{\mu} S\left(y-x^{\prime}\right)=\theta(x)\left[\eta_{c} S\left(x-x^{\prime}\right)\right. \\
& \left.-S\left(x-x^{\prime}\right) \eta_{c}\right]
\end{aligned}
$$

with $x, x^{\prime}$, and $y$ ordinary spacetime coordinates, and $\eta_{c}$ the projection operator defined in (41). Transforming to the momentum space, we end up with the generalization of the first identity of (D1),

$$
S(p+q) Q \eta_{c} S_{F}(p)=i\left[\eta_{c} S(p)-S(p+q) \eta_{c}\right],
$$

Applying the same technique to the integral,

$$
I_{A} \equiv \int_{\mathcal{C}} d^{4} y \frac{\partial \theta}{\partial y_{\mu}} S(x-y) \gamma_{\mu} \gamma_{5} S\left(y-x^{\prime}\right)
$$

we obtain Eq. (42) as the generalization of the second identity of (D1).
[1] V. A. Miransky and I. A. Shovkovy, Quantum field theory in a magnetic field: From quantum chromodynamics to graphene and Dirac semimetals, Phys. Rep. 576, 1 (2015); D. E. Kharzeev, J-f. Liao, S. A. Voloshin, and G. Wang, Chiral magnetic and vortical effects in high-energy nuclear collisions-A status report, Prog. Part. Nucl. Phys. 88, 1 (2016); X. G. Huang, Electromagnetic fields and anomalous transports in heavy-ion collisions-A pedagogical review, Rep. Prog. Phys. 79, 076302 (2016).

[2] D. E. Kharzeev, Parity violation in hot QCD: Why it can happen, and how to look for it, Phys. Lett. B 633, 260 (2006).

[3] D. E. Kharzeev, L. D. McLerran, and H. J. Warringa, The effects of topological charge change in heavy ion collisions: Event by event $\mathcal{P}$ and $\mathcal{C P}$ violation, Nucl. Phys. A803, 227 (2008).

[4] K. Fukushima, D. E. Kharzeev, and H. J. Warringa, The chiral magnetic effect, Phys. Rev. D 78, 074033 (2008).

[5] D. E. Kharzeev and H. J. Warringa, Chiral Magnetic Conductivity, Phys. Rev. D 80, 034028 (2009).

[6] STAR Collaboration, Azimuthal Charged-Particle Correlations and Possible Local Strong Parity Violation, Phys. Rev. Lett. 103, 251601 (2009).

[7] STAR Collaboration, Observation of charge-dependent azimuthal correlations and possible local strong parity violation in heavy ion collisions, Phys. Rev. C 81, 054908 (2010).

[8] STAR Collaboration, Measurement of charge multiplicity asymmetry correlations in high-energy nucleus-nucleus collisions at $\sqrt{s_{N N}}=200 \mathrm{GeV}$, Phys. Rev. C 89, 044908 (2014).

[9] STAR Collaboration, Beam-Energy Dependence of Charge Separation Along the Magnetic Field in
$\mathrm{Au}+\mathrm{Au}$ Collisions at RHIC, Phys. Rev. Lett. 113, 052302 (2014).

[10] ALICE Collaboration, Charge Separation Relative to the Reaction Plane in Pb-Pb Collisions at $\sqrt{s_{N N}}=2.76 \mathrm{TeV}$, Phys. Rev. Lett. 110, 012301 (2013).

[11] Q. Li, D. E. Kharzeev, C. Zhang, Y. Huang, I. Pletikosić, A. V. Fedorov, R. D. Zhong, J. A. Schneeloch, G. D. Gu, and T. Valla, Chiral magnetic effect in $\mathrm{ZrTe}_{5}$, Nat. Phys. 12, 550 (2016).

[12] X-c. Huang et al., Observation of the Chiral-AnomalyInduced Negative Magnetoresistance in 3D Weyl Semimetal TaAs, Phys. Rev. X 5, 031023 (2015).

[13] V. Koch, S. Schlichting, V. Skokov, P. Sorensen, J. Thomas, S. Voloshin, G. Wang, and H.-U. Yee, Chiral magnetic effect task force report, Chin. Phys. C 41, 072001 (2017).

[14] N. Magdy, S-z. Shi, J-f. Liao, N. Ajitanand, and R. Lacey, A new correlator to detect and characterize the chiral magnetic effect, Phys. Rev. C 97, 061901 (2018).

[15] J. Zhao, H-l. Li, and F-q. Wang, Isolating the chiral magnetic effect from backgrounds by pair invariant mass, arXiv:1705.05410.

[16] D-f. Hou, H. Lui, and H-c Ren, Some field theoretic issues regarding the chiral magnetic effect, J. High Energy Phys. 05 (2011) 046.

[17] S. Adler, Axial-vector vertex in spinor electrodynamics, Phys. Rev. 177, 2426 (1969).

[18] S. Adler and W. Bardeen, Absence of higher-order corrections in the anomalous axial-vector divergence equation, Phys. Rev. 182, 1517 (1969).

[19] A. A. Ansel'm and A. A. Iogansen, Radiative corrections to the axial anomaly, Zh. Eksp. Teor. Fiz. 96, 1181 (1989) [Sov. Phys. JETP 96, 670 (1989)]. 
[20] D. Satow and H. U. Yee, Chiral magnetic effect at weak coupling with relaxation dynamics, Phys. Rev. D 90, 014027 (2014).

[21] D. G. Sutherland, Current algebra and some non-strong mesonic decays, Nucl. Phys. B2, 433 (1967); M. Veltman, Theoretical aspects of high energy neutrino interactions, Proc. R. Soc. A 301, 107 (1967).

[22] S. Adler, Anomalies to all orders, arXiv:hep-th/0405040.

[23] M. Peskin and D. Schroeder, An Introduction to Quantum Field Theory (World Scientific, Singapore, 2006).

[24] In this case, $e^{4}$ coming from the QED vertices pertaining to the internal photon lines is replaced by $\frac{1}{2 N_{c}}\left(N_{c}^{2}-1\right) g_{\mathrm{YM}}^{4}$ for the color group $S U\left(N_{c}\right)$ with $g_{\mathrm{YM}}$ the Yang-Mills coupling.

[25] K-c. Chou, Z-b. Su, B-1 Hao, and L. Yu, Equilibrium and nonequilibrium formalisms made unified, Phys. Rep. 118, 1 (1985).

[26] A. Linde, Infrared problem in thermodynamics of the Yang-Mills gas, Phys. Lett. 96B, 289 (1980).

[27] We thank the anonymous referee for reminding us the role of the Debye mass.

[28] S. Coleman and B. Hill, No more corrections to the topological mass term in $\mathrm{QED}_{3}$, Phys. Lett. 159B, 184 (1985).
[29] B. Feng, D-f. Hou, H. Liu, H-c. Ren, P-p. Wu, and Y. Wu, Chiral magnetic effect in a lattice model, Phys. Rev. D 95, 114023 (2017).

[30] V. Voronyk, V. D. Toneev, W. Cassing, E. L. Bratkovskaya, V. P. Konchakovski, and S. A. Voloshin, Electromagnetic field evolution in relativistic heavy-ion collisions, Phys. Rev. C 83, 054911 (2011).

[31] A. Bzdak and V. Skokov, Event-by-event fluctuations of magnetic and electric fields in heavy ion collisions, Phys. Lett. B 710, 171 (2012).

[32] W-t. Deng and X-g. Huang, Event-by-Event generation of electromagnetic field in heavy-ion collisions, Phys. Rev. C 85, 044907 (2012).

[33] D-f Hou, H. Liu, and H-c Ren, A possible higher order correction to the chiral vortical conductivity in a gauge field plasma, Phys. Rev. D 86, 121703(R) (2012).

[34] S. Golkar and D. T. Son, (Non)-Renormalization of the chiral vortical effect coefficient, J. High Energy Phys. 02 (2015) 169.

[35] E. V. Gorbar, V. A. Miransky, I. A. Shovkovy, and X-y Wang, Radiative correction to chiral seperation effect in QED, Phys. Rev. D 88, 025025 (2013). 